\title{
Modulation of fungal virulence through CRZ1 regulated F-BAR-dependent actin remodeling and endocytosis in chickpea infecting phytopathogen Ascochyta rabiei
}

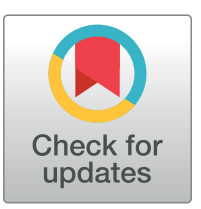

\section{OPEN ACCESS}

Citation: Sinha M, Shree A, Singh K, Kumar K, Singh SK, Kumar V, et al. (2021) Modulation of fungal virulence through CRZ1 regulated F-BARdependent actin remodeling and endocytosis in chickpea infecting phytopathogen Ascochyta rabiei. PLoS Genet 17(5): e1009137. https://doi.org/ 10.1371/journal.pgen.1009137

Editor: Eva H. Stukenbrock, Max-Planck-Institut fur Evolutionsbiologie, GERMANY

Received: September 30, 2020

Accepted: April 13, 2021

Published: May 17, 2021

Peer Review History: PLOS recognizes the benefits of transparency in the peer review process; therefore, we enable the publication of all of the content of peer review and author responses alongside final, published articles. The editorial history of this article is available here: https://doi.org/10.1371/journal.pgen.1009137

Copyright: @ 2021 Sinha et al. This is an open access article distributed under the terms of the Creative Commons Attribution License, which permits unrestricted use, distribution, and reproduction in any medium, provided the original author and source are credited.

Data Availability Statement: All relevant data are within the manuscript and its Supporting Information files.

\author{
Manisha Sinha $\mathbb{D}^{1 \odot}$, Ankita Shree $\mathbb{D}^{1 \oplus}$, Kunal Singh $\mathbb{D}^{10}$, Kamal Kumar $\mathbb{D}^{1}$, Shreenivas \\ Kumar Singh $\mathbb{D}^{1}$, Vimlesh Kumar $\mathbb{C}^{2}$, Praveen Kumar Verma $\oplus^{1,3 *}$ \\ 1 Plant Immunity Laboratory, National Institute of Plant Genome Research, Aruna Asaf Ali Marg, New Delhi, \\ India, 2 Department of Biological Sciences, Indian Institute of Science Education and Research Bhopal \\ (IISER-Bhopal), Bhauri, Bhopal, India, 3 Plant Immunity Laboratory, School of Life Sciences, Jawaharlal \\ Nehru University, New Delhi, India \\ - These authors contributed equally to this work. \\ a Current address: Biotechnology Division, CSIR-Institute of Himalayan Bioresource Technology, Palampur, \\ India \\ * pkv@nipgr.ac.in, praveenkverma@jnu.ac.in
}

\section{Abstract}

Polarized hyphal growth of filamentous pathogenic fungi is an essential event for host penetration and colonization. The long-range early endosomal trafficking during hyphal growth is crucial for nutrient uptake, sensing of host-specific cues, and regulation of effector production. Bin1/Amphiphysin/Rvs167 (BAR) domain-containing proteins mediate fundamental cellular processes, including membrane remodeling and endocytosis. Here, we identified a F-BAR domain protein (ArF-BAR) in the necrotrophic fungus Ascochyta rabiei and demonstrate its involvement in endosome-dependent fungal virulence on the host plant Cicer arietinum. We show that ArF-BAR regulates endocytosis at the hyphal tip, localizes to the early endosomes, and is involved in actin dynamics. Functional studies involving gene knockout and complementation experiments reveal that ArF-BAR is necessary for virulence. The loss-of-function of $A r F-B A R$ gene results in delayed formation of apical septum in fungal cells near growing hyphal tip that is crucial for host penetration, and impaired secretion of a candidate effector having secretory signal peptide for translocation across the endoplasmic reticulum membrane. The mRNA transcripts of $A r F-B A R$ were induced in response to oxidative stress and infection. We also show that ArF-BAR is able to tubulate synthetic liposomes, suggesting the functional role of F-BAR domain in membrane tubule formation in vivo. Further, our studies identified a stress-induced transcription factor, ArCRZ1 (Calcineurinresponsive zinc finger 1), as key transcriptional regulator of $A r F-B A R$ expression. We propose a model in which ArCRZ1 functions upstream of $A r F-B A R$ to regulate $A$. rabiei virulence through a mechanism that involves endocytosis, effector secretion, and actin cytoskeleton regulation. 
Funding: This research received funding from the Core Grant of National Institute of Plant Genome research to PKV., Department of Biotechnology, Government of India, Grant number: BT/PR10605/ PBD/16/791/2008 to PKV, Department of Biotechnology, Government of India, Grant number: BT/AGR/CG-Phase II/01/2014 to PKV and Council of Scientific and Industrial Research, Government of India, Grant number: 19/06/2016(i) EU-V to AS. The funders had no role in study design, data collection and analysis, decision to publish, or preparation of the manuscript.

Competing interests: The authors have declared that no competing interests exist.

\section{Author summary}

BAR-domain superfamily is known to mold amorphous lipid bilayer into defined tubular shapes and required for endosome formation and trafficking. Although these proteins were studied earlier in context of their structural and biochemical properties, there is limited evidence on the direct role of F-BAR domain proteins in the pathophysiological development of other economically important fungi. Our study assumes functional significance for plant infection as we identified an F-BAR domain-containing protein that is regulated by a distinct transcriptional network regulated by a calcium-regulated CRZ1 transcription factor. We characterized ArF-BAR in a necrotrophic fungal pathogen, Ascochyta rabiei, which causes the Ascochyta blight disease of chickpea. Our study will help to understand a signaling cascade that regulates the formation of endosomes, which is required for fungal virulence.

\section{Introduction}

Polarized hyphal growth is a signature feature of filamentous fungi during host colonization [1]. This feature allows the fungus to sense, coordinate, and respond to an array of cues from the host [2]. Therefore, regulation of hyphal tip growth is one of the major virulence determinants in filamentous fungi. In response to infection, the plant innate immune system recognizes pathogen and initiate effective defense response. Pathogens must recognize the plant surface cues and counter host-generated defense responses for effective pathogenesis. Additionally, invasive fungi must overcome an intracellular challenge posed by the distance between the elongated invading hyphal architecture and the nucleus [3]. Mounting evidence strongly suggests that the complications associated with this increased distance are overcome by long-distance intracellular communication for rapid and precise transduction of external information [4]. Moreover, the maintenance of extremely polarized hyphal morphology is heavily dependent on endosome trafficking. It is also important to maintain the structural and functional features of a fungal cell [5].

In the case of filamentous fungi, long-distance signaling is mediated by endosomes, which are multipurpose carriers. Besides signal sensing for motor-dependent retrograde signaling, endosomes are involved in the recycling of cell wall components, polarisome proteins, and various receptors required for polarized tip growth [6]. Loss of these functions leads to impaired host invasion and virulence $[7,8]$. The generation of early endosomes (EEs) is a key step in the endocytic pathway and involves the cooperative action of membrane bending and cytoskeleton reorganization. Membrane bending is the cornerstone for the generation of EEs and is regulated by proteins involved in the detection and stabilization of membrane curvature $[9,10]$.

In animal cells, BAR domain superfamily proteins have been shown to regulate membrane dynamics and remodelling of the actin cytoskeleton [11]. The F-BAR domain proteins form $\mathrm{N}$-terminal $\alpha$-helical coiled-coil dimers and bind to negatively charged lipid membranes via their positively charged residues. This binding generates membrane curvature and regulates intracellular vesicle trafficking [12]. Depending on the degree of curvature in each dimer of BAR proteins, the BAR domain superfamily proteins are broadly classified into three families: classical BAR and N-BAR, Fer/CIP4 homology BAR (F-BAR) and inverse BAR (I-BAR) proteins. The N-BAR and F-BAR domains induce positive membrane curvature through concave lipid-binding interfaces and trigger cell membrane invagination. However, I-BAR domains interact with shallow negatively curved membranes through convex lipid-binding interfaces, leading to cell membrane protrusion $[13,14]$. Pioneering work in the corn smut fungus, 
Ustilago maydis, revealed the importance of endocytosis for the pathogenic development and virulence of filamentous fungi by showing impaired early pathogenicity and germination in endocytic mutants [15]. The F-BAR protein, Cdc15, is involved in cytokinetic ring and septa formation in U. maydis [16]. Recent studies in Magnaporthe oryzae revealed the importance of N-BAR domain-containing proteins in the growth and virulence of filamentous fungi [17]. Further, the I-BAR protein, Rvs167, was found to be involved in the extension of the rigid penetration peg required during M. oryzae invasion [18]. The F-BAR-like protein Bzzlp in yeast and Cip4 in Drosophila act during the early stages of endocytosis in the formation of actin patches $[19,20]$. They trigger actin polymerization via the Arp2/3 complex [21]. There has been an intense study on the structural and biochemical properties of BAR domain proteins that contribute to their mode of action $[11,13]$. However, there has been limited functional characterization of BAR superfamily proteins in phytopathogenic fungi.

Ascochyta rabiei (Pass.) Labr. (teleomorph Didymella rabiei), a causal agent of Ascochyta blight (AB) disease in chickpea (Cicer arietinum $\mathrm{L}$.), is one of the most devastating necrotrophic phytopathogens. A. rabiei infects the above-ground parts of this legume plant and greatly reduces the yield of the crop [22]. The fungal hyphae aggregate in the cortical cells of the chickpea plant and differentiate into asexual spores called pycnidia $[23,24]$. The genome of A. rabiei has been sequenced and analyzed to identify pathogenic determinants [25]. A. rabiei has emerged as an interesting model system for elucidating the cell biology, especially the endocytic machinery, during polar growth and pathogenesis in necrotrophic phytopathogenic fungi.

In this study, a F-BAR domain-containing protein, ArF-BAR, was identified and characterized in A. rabiei. A loss-of-function mutation in ArF-BAR gene cause delayed septa formation, severely compromised fungal virulence on chickpea and secretion of Ar93, which is a candidate effector of $A$. rabiei having a secretory signal peptide sequence. The results showed that the F-BAR domain of the ArF-BAR protein binds to and deforms synthetic liposomes and generates membrane tubules. ArF-BAR transcripts were induced in response to oxidative stress and infection, and the ArF-BAR protein localized to endocytic vesicles within fungal hyphae. It was also found that $A r F-B A R$ expression was regulated by a stress inducible transcription factor, ArCRZ1. Our data suggested that ArF-BAR-dependent actin cytoskeleton dynamics combined with endocytosis and effector secretion is crucial for $A$. rabiei virulence.

\section{Results}

\section{$A r F-B A R$ expression is induced in response to oxidative stress and infection}

Transcriptome analysis of A. rabiei under oxidative stress has provided a greater understanding of the survival strategies used by necrotrophic fungi against host-generated oxidative stress [26]. The study by Singh et al., [26] revealed that 70 unigenes were upregulated under oxidative stress conditions. Of these 70 unigenes, an expressed sequence tag (EST) matched with gene model "ST47_g8005" of the sequenced A. rabiei genome [25] showed early upregulation against oxidative stress. The deduced amino acid sequence of this gene revealed a protein with four distinct domains: An N-terminal F-BAR domain (amino acids 7-253), a unique C1 domain (amino acids 407-459) showing similarity to the $\mathrm{C} 1$ domain of Protein Kinase $\mathrm{C} 1$ (PKC1), and two consecutive C-terminal SH3 domains (amino acids 572-651 and 705-760) (Fig 1A). Henceforth, this protein has been named as ArF-BAR. Phylogenetic analysis of selected BAR proteins from pathogenic fungi and other eukaryotes revealed that ArF-BAR shared sequence identity with proteins of many closely related fungal pathogens (S1 Fig). ArF-BAR was found to share approximately $33 \%$ and $41 \%$ sequence identity with 
A

\begin{tabular}{|l|l|l|l|}
\hline F-BAR & C1 & SH3a & SH3b-C \\
\hline
\end{tabular}

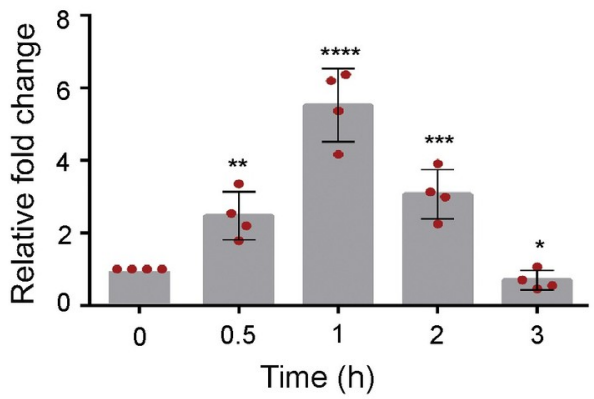

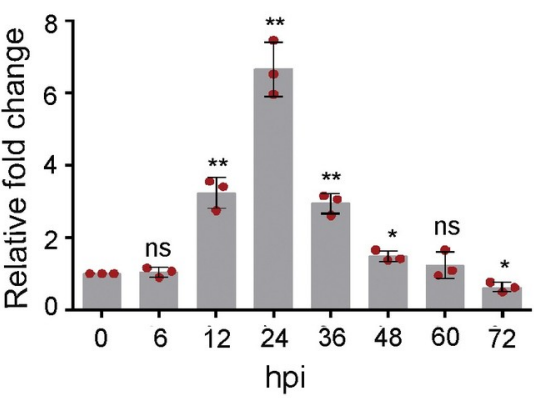

Liposomes supplemented with

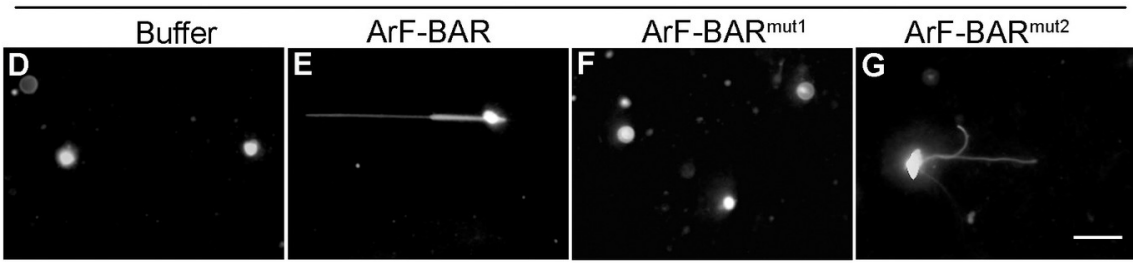

H

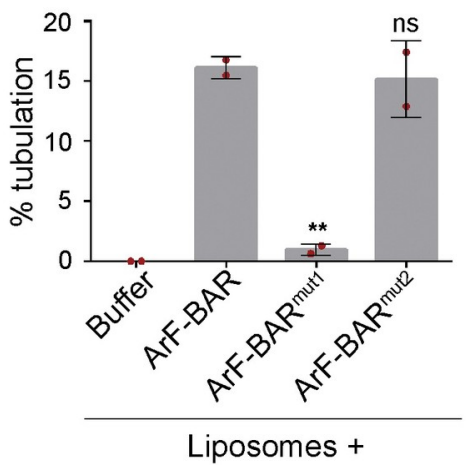

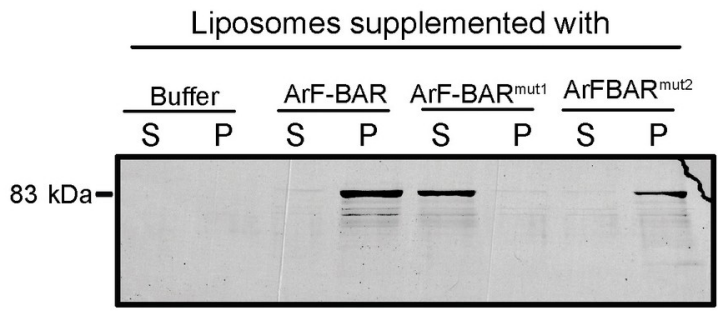

Fig 1. ArF-BAR is a bonafide stress induced membrane tubulating protein. Schematic representation of domain organization of ArF-BAR protein. It has a F-BAR domain (amino acids 7-253), a C1 domain of Protein Kinase C1, PKC1 (amino acids 407-456) and two consecutive SH3 domains (SH3a; amino acids 572-651 and SH3b; amino acids 705-760). (B) Relative fold change in the $A r F-B A R$ transcript level of 5 days axenically grown potato dextrose broth mycelium culture of Ascochyta rabiei post $250 \mu \mathrm{M}$ menadione treatment. The treated mycelia samples were collected every $0.5 \mathrm{~h}$ and the expression was analysed with qRT-PCR. (C) Relative fold change in the transcript level of $A r F-B A R$ post $A$. rabiei conidial suspension inoculation. The conidia inoculated chickpea aerial tissue was collected up to $72 \mathrm{hpi}$ and expression was analysed by qRT-PCR. (D-G) Membrane deforming activity of ArF-BAR. The bar represents $20 \mu \mathrm{m}$. (D) The liposomes remain spherical in presence of protein resuspension buffer, acting as control. (E, G) Tubulation of synthetic liposomes after addition of the recombinant ArF-BAR and ArF-BAR ${ }^{\text {mut } 2}$ proteins. (F) Liposome tubulation activity is insignificant in ArF-BAR ${ }^{\text {mut1 }}$. (H) The bar chart shows the percentage of the tubule formed by liposomes in presence of native and mutated recombinant proteins. Out of 150 liposomes, the percentage of the tubule formation was counted in each set of experiment. (I) Liposome co-sedimentation assay, where the ArF-BAR and ArF-BAR ${ }^{\text {mut2 }}$ recombinant protein bound with lipid membranes (liposome) were found in pellet, while 
membrane binding activity is lost in ArF-BAR ${ }^{\text {mut } 1}$ protein. Statistical analysis was performed using Student's t-test one tailed compared with its control. Significant differences are indicated as ${ }^{*} p=0.03 ;{ }^{* *} p=0.003 ;{ }^{* * *} p=0.0005$; ${ }^{* * * *} p=0.0001 ; \mathrm{ns}=$ non-significant. Data is the mean of three independent biological replicates with error bars \pm representing standard deviation. Red dots represent the average value of all technical replicate in each biological replicate used for the quantitative analysis.

https://doi.org/10.1371/journal.pgen.1009137.g001

Saccharomyces cerevisae BZZ1p and Drosophila melanogaster Cdc42-Interacting Protein 4 (CIP4) proteins, respectively (S2 Fig).

To validate the results of the transcript profiling, quantitative real-time PCR (qRT-PCR) was performed using primers specific to the F-BAR domain encoding region. The qRT-PCR experiments revealed a higher expression level of the ArF-BAR transcript in A. rabiei mycelia after a $1 \mathrm{~h}$ treatment with menadione, which is an oxidative stress generator (Fig 1B). To directly assess $A r F-B A R$ transcript induction during pathogen proliferation on a susceptible chickpea variety (PUSA-362), the time course of $A r F-B A R$ transcript expression was measured using qRT-PCR after inoculating plants with $A$. rabiei conidial suspension. Consistent with the previous observations [26], a significant increase in the $A r F-B A R$ transcript level was found following infection. The maximum transcript level was at 24 hours post-inoculation (hpi; Fig 1C), which is within the critical time period for spores to germinate on the host surface and early germinated conidial hyphae starts penetrating chickpea tissue [24,27].

\section{ArF-BAR is a membrane tubulating protein}

To understand whether ArF-BAR has a functional F-BAR domain, synthetic liposomes containing Rhodamine B-conjugated $\mathrm{PE}$ were incubated with purified His-tagged recombinant ArF-BAR protein (S3A Fig). Narrow tubules were formed after 10 min of liposomes incubation with purified recombinant ArF-BAR protein (Fig 1D and 1E). The spherical liposomes transformed into an intense network of narrow tubules within 30 min of incubation with ArF-BAR (S3B Fig). This indicated that ArF-BAR protein has liposome tubulation activity. Additionally, the sequence alignment of ArF-BAR with that of other characterized F-BAR domain-containing proteins, revealed the presence of positively charged conserved residues (S2 Fig). In previous studies, the positively charged residues of FBP17 and Syndapin have been shown to interact with negatively charged phospholipids to induce membrane curvature $[28,29]$. Since ArF-BAR has conserved positively charged amino acids at positions 57, 58, 131 and 132 (S2 Fig), the role of these residues in membrane deformation was evaluated. This evaluation was performed by replacing the residues with glutamate (K57E, K58E, R131E and $\mathrm{K} 132 \mathrm{E})$ via site-directed mutagenesis, and the mutant version was referred to as ArF-BAR ${ }^{\text {mut1 }}$ (S3C Fig). As expected, it was found that substituting the conserved lysine/arginine with glutamate abolished the tubulation activity of the F-BAR domain (Fig 1F). Protein kinase C1 (PKC1) proteins are known to interact with diacylglycerol (DAG), which has a role in membrane interactions. To abolish the involvement of the unique $\mathrm{C} 1$ domain of ArF-BAR in membrane deformation, residues at positions W428 and L430 were replaced with glycine [30], and the mutant form was referred to as ArF-BAR ${ }^{\text {mut2 }}$ (S3D Fig). Further, this ArF-BAR ${ }^{\text {mut2 } 2}$ protein was used to assess the in vitro liposome tubulation activity and a similar tubulation activity to that of the wild-type ArF-BAR protein was found (Fig 1G). Thus, positively charged amino acids of F-BAR domain are involved in ArF-BAR mediated membrane tubulation (Fig 1H). To test whether the tubulation activity of ArF-BAR correlated with its lipid binding ability, a liposome co-sedimentation assay was used (Fig 1I). Compared to the native ArF-BAR protein, ArF-BAR ${ }^{\text {mut } 1}$ showed reduced lipid sedimentation efficiency. In contrast, the sedimentation was unaffected with ArF-BAR ${ }^{\text {mut2 }}$. This indicated the importance of the direct interaction of 
the BAR domain with the lipids in liposome tubulation (Fig $1 \mathrm{H}$ and 1I). Together, these results suggest that F-BAR domain of ArF-BAR plays a major role in interacting with the lipid membranes for binding and deformation.

\section{ArF-BAR is required for A. rabiei virulence on chickpea}

To elucidate the biological importance of $A r F-B A R$ in fungal virulence, an A. rabiei knockout mutant ( $\triangle a r f-b a r)$ was generated. The $A r F-B A R$ gene was targeted for deletion using a homologous recombination approach. The results confirmed that the open reading frame (ORF) of $A r F-B A R$ had been successfully replaced with a single copy of the hygromycin resistance gene (Hph; S4A and S4B Fig). Simultaneously, $\triangle a r f-b a r$ mutant strain was complemented with a T-DNA cassette that expressed ArF-BAR under its own promoter ( $\triangle a r f-b a r / A r F-B A R$; S5A and S4B Figs).

To assess the effect of $A r F-B A R$ deletion on vegetative growth of $A$. rabiei; wild-type (WT), $\triangle a r f-b a r$, and knockout complemented ( $\triangle a r f-b a r / A r F-B A R)$ strains were grown on potato dextrose agar (PDA) plates. The knockout mutants showed reduced radial growth compared to $A$. rabiei wild-type (WT) and this radial growth was restored in the $\triangle a r f-b a r / A r F-B A R$ (Fig 2A). To determine the Ascochyta blight $(\mathrm{AB})$ disease development and virulence of the fungal strains, conidial suspensions of each strain were spray inoculated on susceptible chickpea variety plants individually. The typical $\mathrm{AB}$ disease symptoms, described previously $[23,24]$, were observed on chickpea plants challenged with the A. rabiei wild-type (WT) and $\triangle a r f-b a r / A r F-$ $B A R$, but not on plants challenged with $\triangle a r f-b a r$ (Fig 2B). The degree of pathogenicity was quantified according to lesion number and lesion size, which were compared among the $A$. rabiei wild-type (WT) and mutants at $144 \mathrm{hpi}$. The lesion number per plant in $\Delta a r f-b a r$ was significantly lower than in the A. rabiei wild-type (WT) (Fig 2C). The mean lesion size was also much lower in $\triangle a r f-b a r$ than in the A. rabiei wild-type (WT) (Fig 2D). However, for the $\triangle a r f-b a r / A r F-B A R$ complementation mutant, the disease symptoms were comparable to those of the A. rabiei wild-type WT. Overall, the disruption of the ArF-BAR gene significantly impaired the pathogenicity of $A$. rabiei.

To ascertain the role of F-BAR and unique $\mathrm{C} 1$ domains in fungal virulence, the two domains were independently inactivated by site-directed mutagenesis as described earlier. The $\Delta a r f$-bar knockouts were complemented with residues mutated in F-BAR domain ( $\triangle a r f-b a r$ ) $A r F-B A R^{\text {mut } 1}$; S5B and S4B Figs) and C1 domain ( $\triangle$ arf-bar/ArF-BAR ${ }^{\text {mut2 }}$; S5C and S4B Figs). In three independent biological replicates, each replicate having at least 20 plants, the WT strain produced 5.6 times greater number of disease lesions per plant in comparison those produced by $\Delta$ arf-bar mutant strain (WT $=14.99 \pm 0.84 ; \Delta$ arf-bar $=2.66 \pm 0.19 ; p=0.000536$; Fig $2 C$ ). As compared to the WT, the virulence of $\triangle a r f-b a r / A r F-B A R^{\text {mut1 }}$ strain was very low (disease lesions/plant $=3.02 \pm 0.74$; mean lesion size $=1.33 \mathrm{~mm} \pm 0.06$ ). Meanwhile, in the case of $\triangle a r f-b a r / A r F-B A R^{\text {mut2 }}$ strain the number of lesions per plant was significantly lower $(8.833 \pm 0.04 ; p=0.000127)$ than in the WT, but there was no significant difference in the size of the lesions (WT $=3.67 \mathrm{~mm} \pm 0.46 ; \Delta$ arf-bar $/ A r F-B A R^{\mathrm{mut} 2}=2.97 \mathrm{~mm} \pm 0.15$; Fig $2 \mathrm{D}$ ). The severity of the disease symptoms increased in the plants after 10 days post infection (dpi), but no significant differences were observed for plants challenged with $\triangle a r f$-bar strain (S6 Fig). Together, these results confirm that $A r F-B A R$ is an important virulence determinant and that the conserved positively charged residues of F-BAR domain are required for full-virulence of A. rabiei on chickpea.

It was subsequently hypothesized that the reduced virulence in $\Delta a r f-b a r$ could have resulted from at least two factors: a) knockout mutants may have had a compromised ability to penetrate host tissue, or b) the reduced virulence of the mutant was a consequence of a reduction in 
A

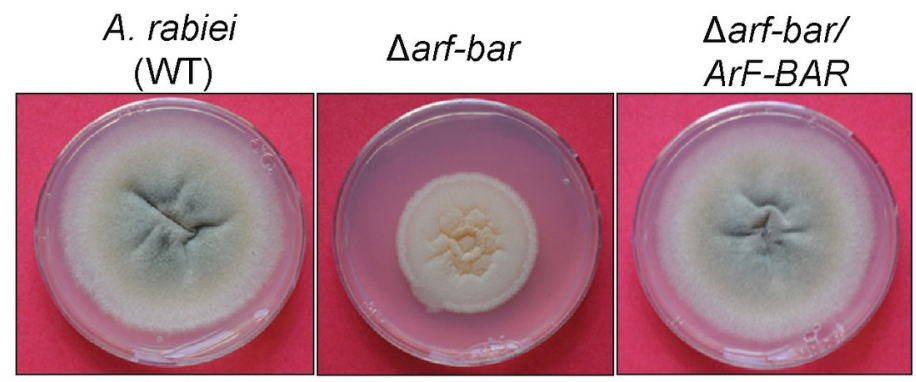

B

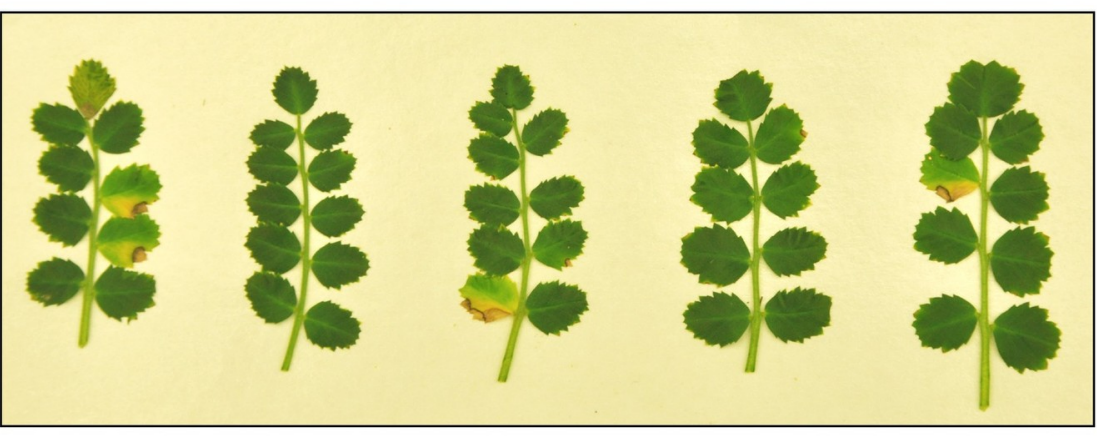

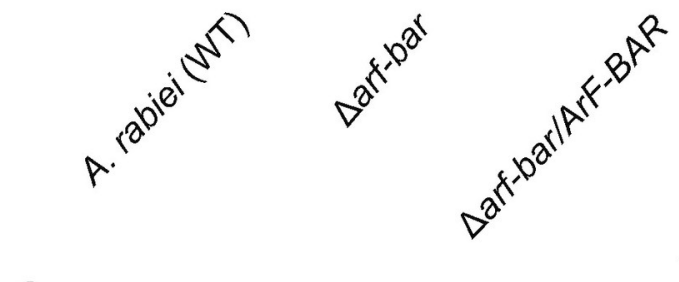

C

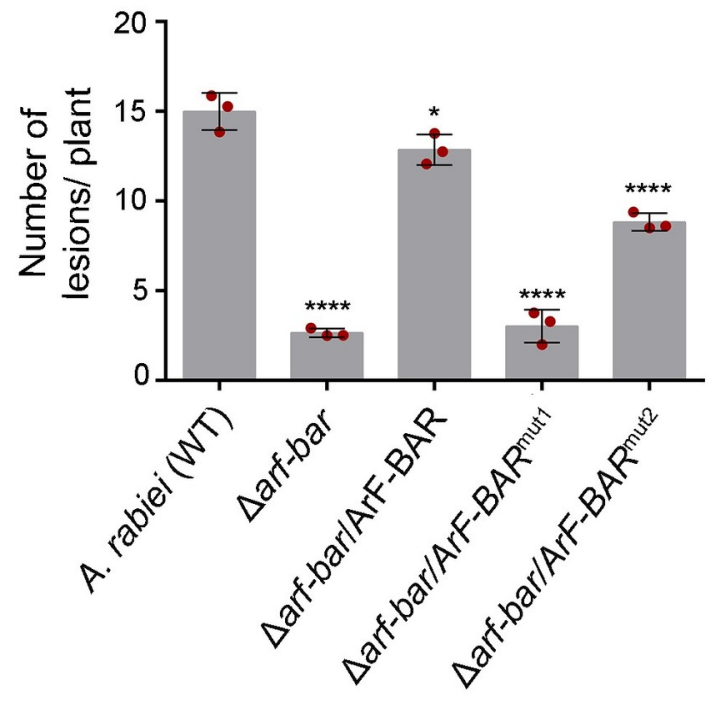

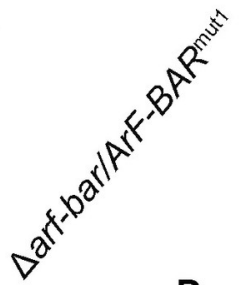

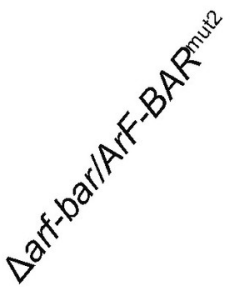

D

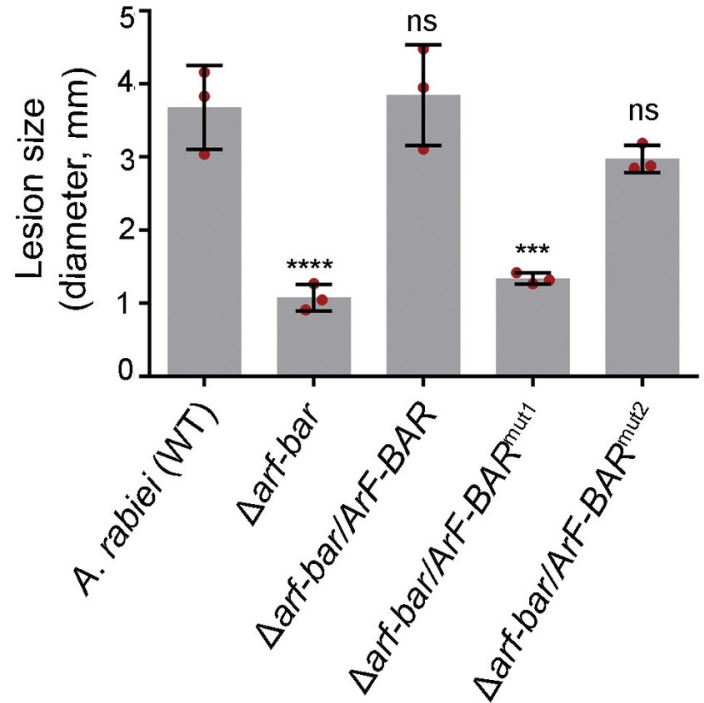

Fig 2. ArF-BAR is required for full virulence of $A$. rabiei in chickpea. (A) To examine radial vegetative growth on PDA plates, $A$. rabiei (WT), $\triangle a r f-b a r$ and $\triangle a r f-b a r / A r F-B A R$ mutant strains were grown for 7 days at $22^{\circ} \mathrm{C}$. (B) Representative image of Ascochyta blight $(\mathrm{AB})$ disease symptoms on susceptible chickpea plants at $6 \mathrm{dpi}$, obtained after inoculation with conidial suspension of $A$. rabiei (WT) and mutant strains. Decreased AB symptoms in $\triangle a r f-b a r$ and $\triangle a r f-b a r / A r F-B A R^{\text {mutl }}$ shows reduced virulence. (C) Statistical values of the number of lesions per plant after conidial inoculation of chickpea with A. rabiei (WT), mutant and mutant complemented strains. The graph represents the mean and standard deviation of three independent biological replicates, counting at least 20 plants in each replicate. (The bars in graph correspond to $14.99 \pm 0.84$ in WT; $2.66 \pm 0.19$ in $\triangle$ arf-bar; $12.86 \pm 0.69$ in $\triangle$ arfbar/ArF-BAR; $3.02 \pm 0.74$ in $\triangle a r f-b a r / A r F-B A R^{\text {mut1 }}$ and $8.833 \pm 0.04$ in $\triangle a r f-b a r / A r F-B A R^{\text {mut2 }}$ ). (D) Statistical values of the lesions' 
diameter were calculated (The bar in graphs corresponds to; $\mathrm{WT}=3.67 \mathrm{~mm} \pm 0.46 ; \Delta \operatorname{arf}-$ bar $=1.07 \mathrm{~mm} \pm 0.14 ; \Delta \operatorname{arf}-$ bar $/ A r F-B A R=$ $3.84 \pm 0.54 ; \triangle a r f-b a r / A r F-B A R^{\text {mut1 }}=1.33 \pm 0.06$ and $\left.\Delta a r f-b a r / A r F-B A R^{\text {mut2 }}=2.97 \pm 0.15\right)$. Statistical analysis was performed using ordinary one-way ANOVA compared with its control $\left({ }^{* * * *} p<0.0001 ;{ }^{* * *} p<0.001 ;{ }^{*} p<0.05\right)$. Red dots represent the average value of all technical replicate in each biological replicate used for the quantitative analysis.

https://doi.org/10.1371/journal.pgen.1009137.g002

fungal viability or inability to counter the host basal defense. To address these possibilities, hyphal penetration was examined and compared by inoculating chickpea leaves with the WT and $\Delta a r f$-bar mutant strains. The leaves of the chickpea plants challenged with these strains were subjected to wheat germ agglutinin-Alexa Fluor conjugate (WGA-AF488) staining. At 48 hpi, the infected leaves were stained with WGA-AF488 to enable visualization of the fungus [31]. The infected leaves were optically sectioned using confocal microscopy starting from the surface of the leaves. It was consistently observed that hyphae of the A. rabiei wild-type (WT) strain efficiently penetrated into the chickpea leaves up to depths of $20.25 \mu \mathrm{m} \pm 3.92$ (mean \pm $\mathrm{SD} ; \mathrm{n}=6$ ) (S7A and S7C Fig). In stark contrast, the hyphae of the $\Delta$ arf-bar mutant were unable to penetrate beyond $9.83 \mu \mathrm{m} \pm 2.54$ ( $p=0.0079$; S7B and S7C Fig). Thus, the depth and efficiency of hyphal penetration by $\triangle a r f-b a r$ were significantly impaired.

Further, to investigate the direct involvement of $A r F-B A R$ in fungal viability under oxidative stress conditions, radial growth assays were performed for WT, $\Delta a r f-b a r, \Delta a r f-b a r / A r F-$ $B A R, \triangle a r f-b a r / A r F-B A R^{\text {mut1 }}$, and $\triangle a r f-b a r / A r F-B A R^{\text {mut2 }}$ strains. These strains were inoculated on either potato dextrose agar (PDA) or PDA supplemented with menadione $(250 \mu \mathrm{M}$ and $500 \mu \mathrm{M}$ ) or $2 \mathrm{mM} \mathrm{H}_{2} \mathrm{O}_{2}$. The radial growth was analyzed at $10 \mathrm{dpi}$. The radial growth of $\Delta a r f-$ bar was reduced compared to that of the WT. The mycelial growth was restored in the wildtype $A r F-B A R$ complemented ( $\triangle a r f-b a r / A r F-B A R)$ and $A r F-B A R^{\text {mut2 }}$ complemented $(\Delta a r f-$ bar/ArF-BAR ${ }^{\text {mut2 }}$ ) strains (Fig $3 \mathrm{~A}$ and $3 \mathrm{~B}$ ). However, the mycelial growth of complemented $\triangle a r f-b a r / A r F-B A R^{\text {mutl }}$ was unable to match that of the WT. Exposure to oxidative stress led to further growth inhibition in $\triangle a r f-b a r$ (Fig 3). The phenotype of complemented strain, $\Delta a r f-$ bar/ArF-BAR, under oxidative stress suggest that cellular processes regulated by ArF-BAR are important for oxidative stress tolerance (Fig 3). Further, exposure to oxidative stress led to greater growth inhibition in $\triangle a r f-b a r / A r F-B A R^{\text {mut1 }}$ than in the wild-type. However, the growth inhibition of $\triangle a r f-b a r / A r F-B A R^{\text {mut2 }}$ was similar to that of wild-type. The negative effect on growth of fungal strains till $10 \mathrm{dpi}$ was more pronounced for $\mathrm{H}_{2} \mathrm{O}_{2}$ as compared to menadione (Figs 3 and S8). These results strongly support the hypothesis that $A r F-B A R$ is required for full virulence of $A$. rabiei wild-type (WT) on susceptible chickpea host and is required for coping with the host basal defense, mainly oxidative stress.

\section{Absence of ArF-BAR perturbs septa formation}

Having a potential role in fungal virulence, the next aim was to determine the subcellular localization of ArF-BAR inside A. rabiei wild-type (WT) hyphal cells. The ectopic expression of EYFP decorated ArF-BAR chimeric protein was achieved by Agrobacterium tumefaciensmediated transformation (ATMT) of ArF-BAR:EYFP T-DNA cassette in $\triangle$ arf-bar strain [27]. The bioimaging of the enhanced yellow fluorescent protein tagged ArF-BAR showed punctate distribution throughout the cytoplasm of the fungal hyphae. The chimeric protein was mostly concentrated at the growing hyphal tip and at the septa (Fig 4A and 4B). Fungal hyphae transformed with chimeric ArF-BAR ${ }^{\text {mut } 1}::$ EYFP exhibited disrupted localization of these punctate structures and the fluorescence was completely diffused throughout the cytoplasm. However, the distribution of the fluorescent puncta was unaffected in hyphae of strains stably expressing ArF-BAR ${ }^{\text {mut2 }}:$ :EYFP (Fig 4A). In this strain, the punctate structures were distributed throughout the cytoplasm and were prominently concentrated at the growing hyphal tip (Fig 4A). 
A

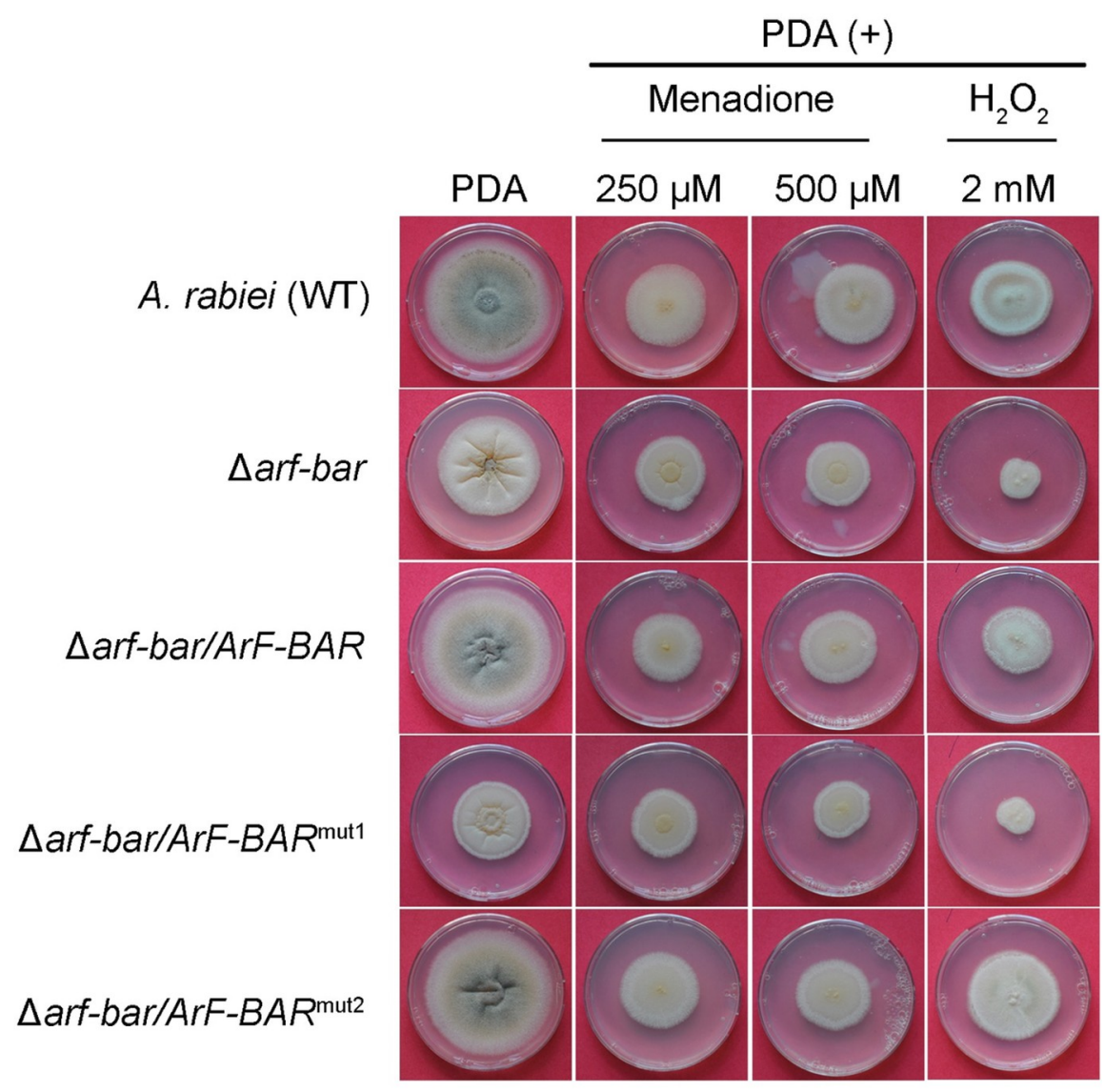

B

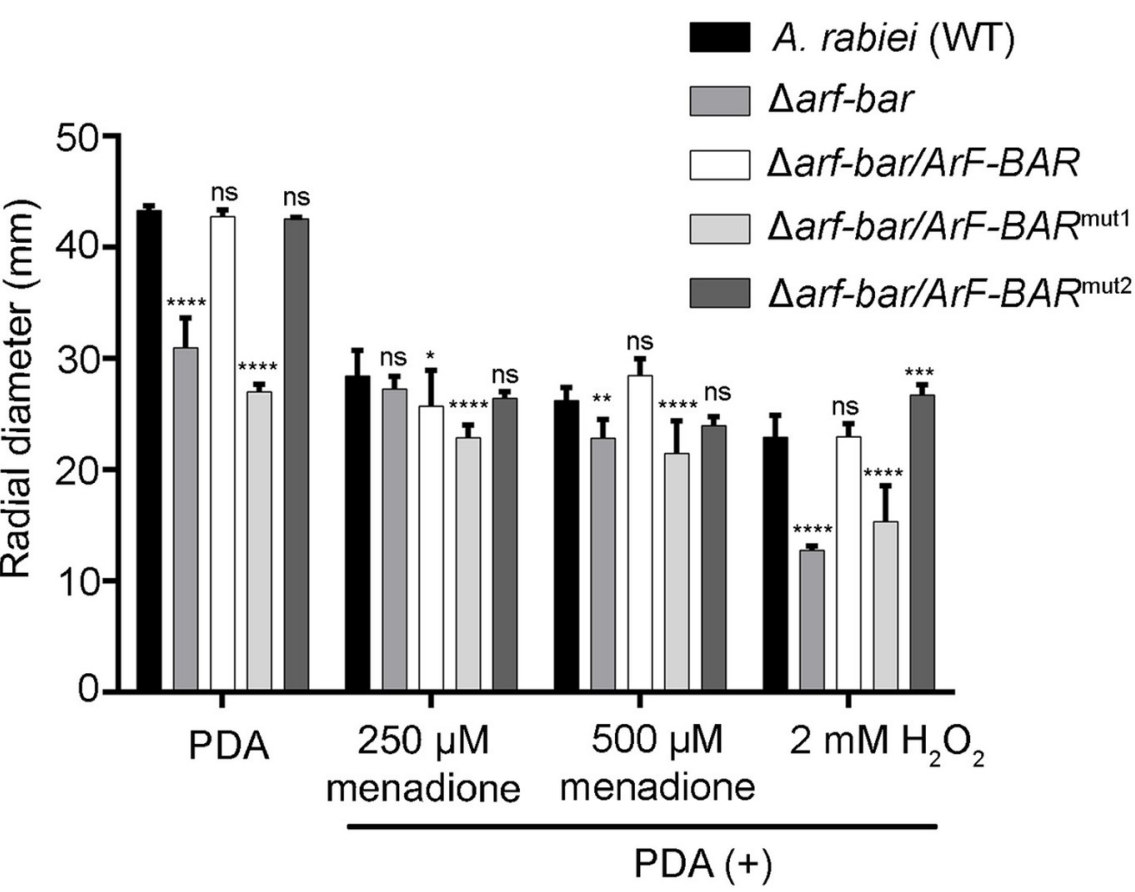


Fig 3. $A r F-B A R$ is required for vegetative growth and oxidative stress tolerance. (A) Colony morphology and growth assay of A. rabiei (WT), $\Delta a r f-b a r$ mutant and mutant complemented strains, observed 10 days after incubation at $22^{\circ} \mathrm{C}$. For oxidative stress, the PDA medium was supplemented with $250 \mu \mathrm{M}$ and $500 \mu \mathrm{M}$ menadione, and $2 \mathrm{mM}$ $\mathrm{H}_{2} \mathrm{O}_{2}$. Strains of $\triangle a r f$-bar and $\triangle a r f-b a r / A r F-B A R^{\text {mut }}$ exhibited enhanced sensitivity towards oxidative stress condition as compared to WT A. rabiei. (B) The bar graph represents mean diameter and SD values of fungal strains radial growth in PDA plates and PDA supplemented with menadione or $\mathrm{H}_{2} \mathrm{O}_{2}$. All the PDA growth assays were performed in triplicate. The results were quantified using two-way ANOVA, Tukey's multiple comparisons. ${ }^{* * * *} p<0.0001$, ${ }^{* * *} p<0.001,{ }^{* *} p<0.01,{ }^{*} p<0.05, \mathrm{~ns}=$ non-significant.

https://doi.org/10.1371/journal.pgen.1009137.g003

Further, to validate the spatiotemporal distribution pattern of the ArF-BAR protein during infection, fungal spores expressing ArF-BAR::EYFP were allowed to infect susceptible chickpea stem peel. Microscopic observation of these stem peel infected with fungal hyphae revealed a distribution pattern that was similar to that of the ArF-BAR::EYFP expressing hyphae growing on glass slides; the chimeric protein was predominantly distributed at the hyphal tip and septa (S9 Fig). The localization of ArF-BAR::EYFP towards the membrane of growing hyphae prompted us to check its localization with a Spitzenkörper marker ArSNC1, which is a vesiclebound v-SNARE protein known for its role in polarized exocytosis in conjugation with other proteins [32-34]. ArSNC1 was identified, from A. rabiei genome, based on its high homology with the M. oryzae SNC1 (MGG_12614.6) encoded protein. The localization pattern of ArF-BAR::EYFP and ArSNC1::mCherry confirms the apical localization of ArF-BAR (Fig 4C). The in vitro germination and growth of WT and $\Delta a r f$-bar conidia, up to $12 \mathrm{~h}$ on hydrophobic surface showed marginal difference (S10 Fig). However, the first cell from the growing hyphal tip in $\triangle a r f$-bar was observed to be elongated as compared to the WT, which may be due to delayed septation in $\triangle a r f-b a r$ as ArF-BAR localizes to the septa. The arrangement of septa within the hyphae of both the WT and the $\Delta a r f-b a r$ strain was examined in a number of growing fungal tips. Microscopic analysis of the $\Delta a r f$-bar mutant using calcofluor white, which precisely stains cell wall components, showed that the hyphae lacked regularly spaced septa (Fig 5B). Interestingly, the distance of the first septum from the growing hyphal tip (polarized end) was significantly greater in the $\Delta$ arf-bar mutant than in the WT (WT $=23.5 \mu \mathrm{m} \pm 3.23$ and $\Delta a r f-b a r=$ $58.5 \mu \mathrm{m} \pm 4.65 ;$ mean \pm SEM; $n=40$ and $p<0.0000496$; Fig 5 C). It showed that $\Delta a r f-b a r$ mutant has larger cell length of first and second cells, from the hyphal tip, as compared to $A$. rabiei wild-type (WT) (Fig 5C). Overall, these results reveal that the ArF-BAR gene is necessary for appropriate hyphal architecture, which is in turn, important for host penetration and virulence.

\section{The $\Delta a r f$-bar mutant impairs secretion of a candidate effector from $A$. rabiei}

The delayed proliferation of $\Delta a r f$-bar strain compared to that of the WT, on susceptible plant surface and less penetration of host tissue (Figs 5A and S7) points towards mutant strain's defect in normal development or suppression of host defense. Therefore, the substantial reduction in virulence of $\Delta a r f-b a r$ on chickpea led us to hypothesize that the deletion mutant may be impaired in the ability to secrete effectors compared to A. rabiei wild-type (WT). To confirm the likely role of ArF-BAR in effector secretion, a candidate secretory effector Ar93 (Accession number: GW996416) [26] possessing a secretory signal peptide for secretion was ectopically expressed in WT and $\Delta a r f$-bar backgrounds. The secretion of Ar93 protein fused with EYFP::FLAG to the culture media was examined using concentrated proteins from culture filtrate and cell lysates of WT and $\Delta a r f-b a r$. Western blots analysis of culture filtrate of axenically grown stable fungal transformants (WT and $\Delta$ arf-bar) showed exclusive secretion of Ar93 effector only in WT transformed strain. However, Ar93 was observed in cell lysates of 
A

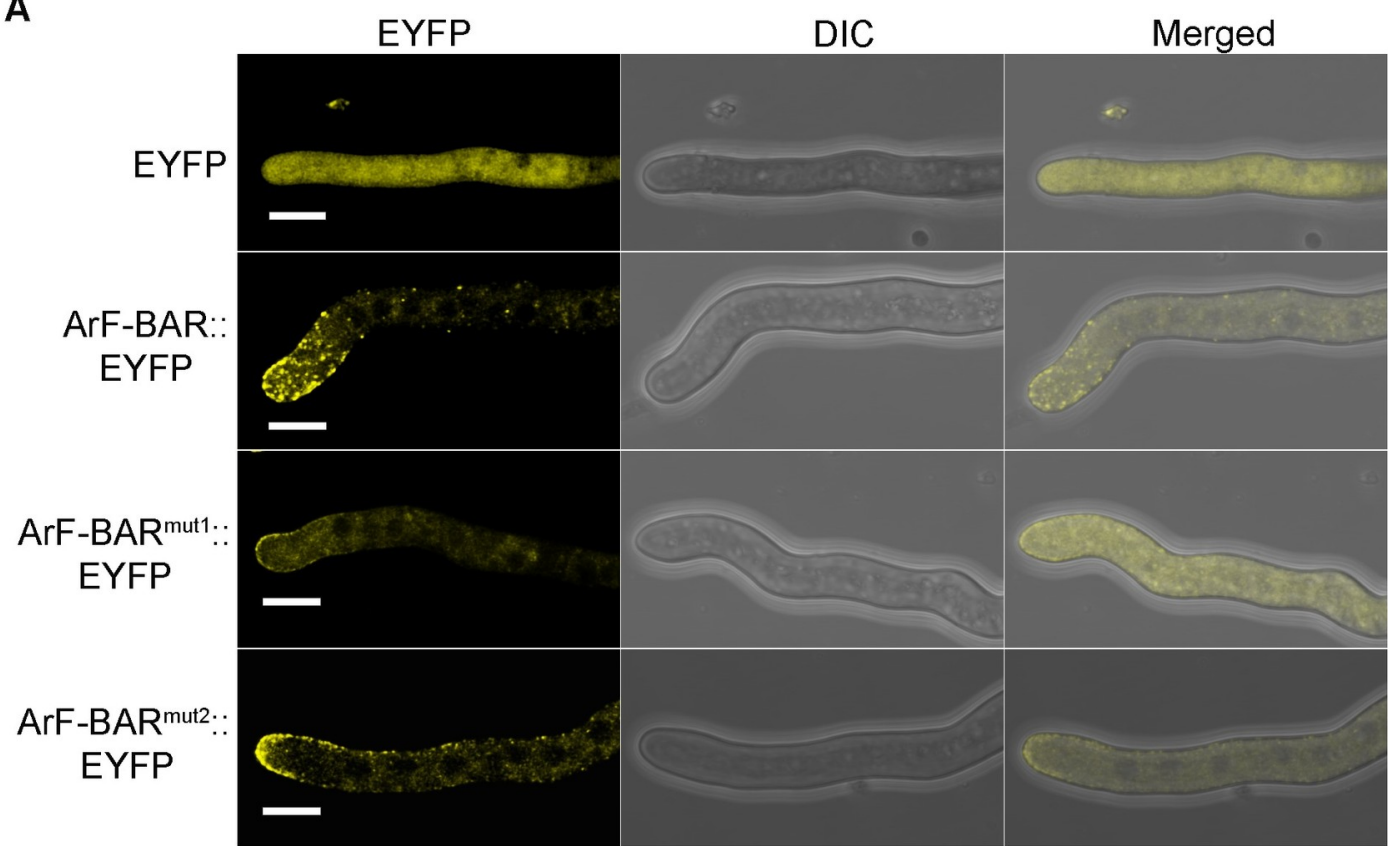

B

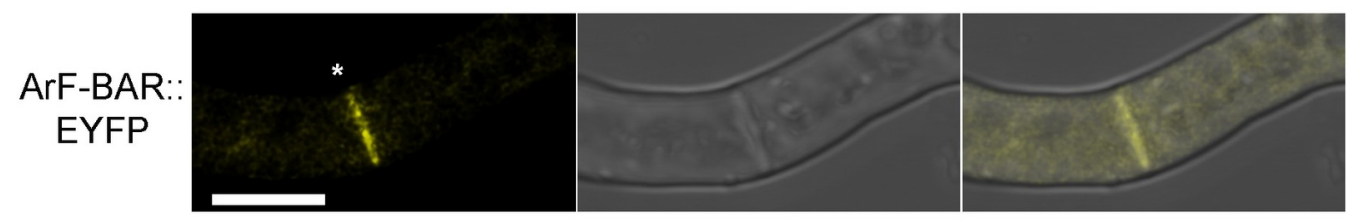

C

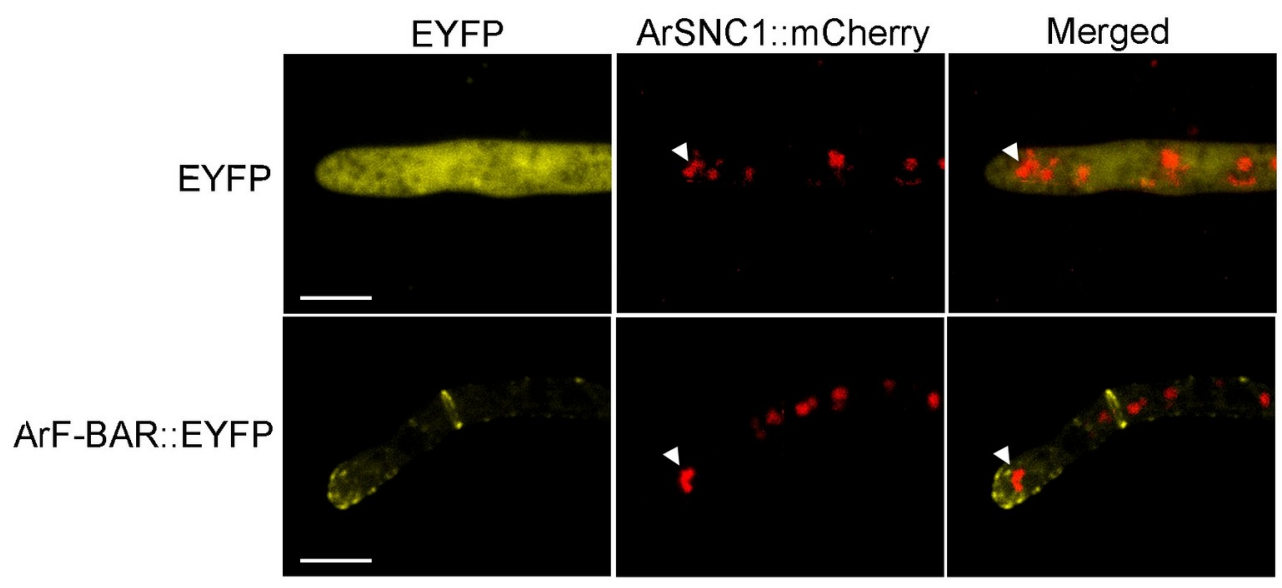

Fig 4. ArF-BAR primarily decorates the growing hyphal tip. (A) Ectopically expressed ArF-BAR::EYFP and ArF-BAR ${ }^{\text {mut }^{2}::}$ EYFP chimeric proteins are distributed at the hyphal tip. Fungal cells expressing ArF-BAR ${ }^{\text {mut }}:: E Y F P$ had fluorescence scattered throughout the fungal hyphae. The representative images were captured at $12 \mathrm{~h}$ post germination of fungal conidia on microscopic coverslip. Scale bar $=5 \mu \mathrm{m}$. (B) ArF-BAR::EYFP signal is uniformly distributed at fungal septum, scale bar $=5 \mu \mathrm{m}$. Star represents the hyphal septum. (C) The growing hyphal tip localization of ArF-BAR::EYFP was examined along with $A$. rabiei Spitzenkörper marker, ArSNC1::mCherry. Arrowhead represents the localization of ArSNC1. The v-SNARE SNC1 along with other proteins mediate docking and fusion of vesicles with the plasma membrane target site. Micrographs show representative images of three biological replicates with at least 10 images in each replicate.

https://doi.org/10.1371/journal.pgen.1009137.g004 
A
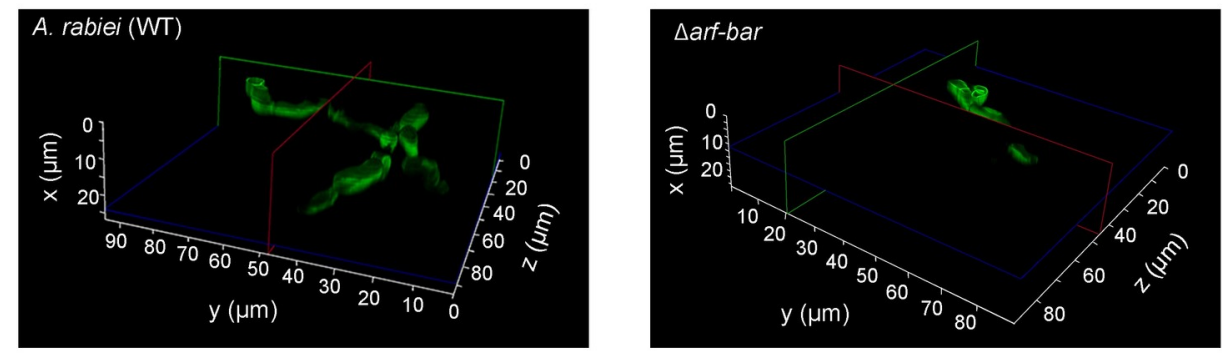

B

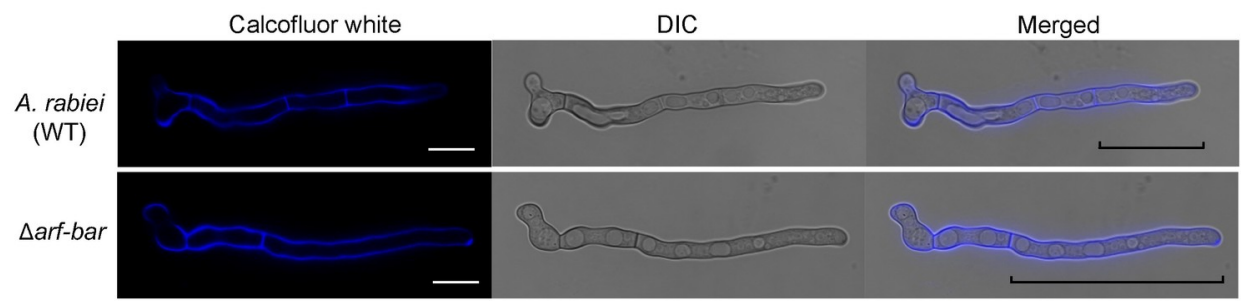

C

D
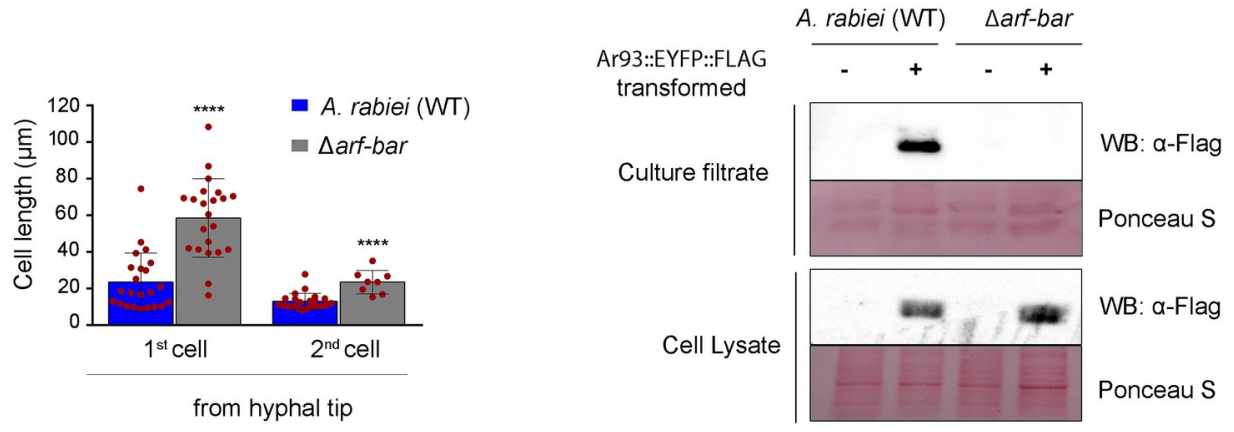

Fig 5. Deletion of $A r F-B A R$ gene perturbs septum formation, host penetration and effector secretion. (A) 3D structure stacking $\mathrm{z}$-slices together shows the penetration ability of $A$. rabiei (WT) and $\Delta$ arf-bar on the chickpea host leaves. The $\Delta$ arf-bar mutant could penetrate on an average $Z$ value of $10 \mu \mathrm{m}$ as compared to WT value of $20 \mu \mathrm{m}$. (B) Calcofluor white stained A. rabiei (WT) and $\Delta a r f-b a r$ hyphae after $12 \mathrm{~h}$ post germination. Increase in the distance of first septa from the polarised end was found in $\Delta a r f$-bar. Black line marks the position of first septa from growing hyphal tip in both WT and $\Delta a r f-b a r$. Scale bar $=10 \mu \mathrm{m}$. (C) Bars in graph represent the mean values with SEM, of first cell and second cell length (in $\mu \mathrm{m}$ ) from the growing tip end. Significance of the difference in length of cells was calculated using one-tailed paired t-test, $\left({ }^{* * * *} p=0.001\right)$. Red dots represent the value of each biological replicate used for the quantitative analysis. (D) Secretion of an ectopically expressed candidate secretory effector possessing a classical signal peptide, Ar93::EYFP::Flag (43 kDa) in WT and $\Delta$ arf-bar mutant. The $\Delta a r f-b a r$ strain of $A$. rabiei is impaired in effector secretion in contrast to WT strain. The absence of signal in the western blot in $\Delta a r f-b a r$ mutant represents absence of secreted Ar93 effector in culture filtrate, although the chimeric Ar93 is present in the cell lysate of both strains. The minus (-) sign represents Ar93 non-transformed and plus (+) represents Ar93:EYFP:FLAG transformed $A$. rabiei strains. Ponceau S stained blots represent protein loading control.

https://doi.org/10.1371/journal.pgen.1009137.g005

both the WT and $\Delta a r f$-bar suggesting Ar93 protein expressed in both strains (Fig 5D). Thus, we conclude that $\Delta a r f-b a r$ mutant is impaired in secretion of Ar93 or similar effectors possessing classical secretory signal peptide.

\section{The $\Delta a r f$-bar mutant has impaired endocytosis}

Since F-BAR domain proteins are known to dimerize and form a canonical banana-shaped fold [35], the dimerization of ArF-BAR was confirmed using a spilt-ubiquitin based yeast two- 
hybrid $(\mathrm{Y} 2 \mathrm{H})$ system. The growth of yeast cells on selective media and expression of $\beta$-gal reporter gene suggested that ArF-BAR protein dimerize. This evidence further indicates the evolutionarily conserved nature of the BAR proteins (S11 Fig). To gain insight into the role played by ArF-BAR in endocytosis, an amphiphilic styryl type endocytic tracer dye, N(3-triethylammoniumpropyl)-4-(p-diethyl-aminophenyl-hexatrienyl) pyridiniumdibromide (FM4-64), was used [36]. Microscopic analysis of FM4-64 stained WT and Darf-bar hyphae revealed internalization of the dye in WT but not in mutant cells. The results suggested rapid internalization of dye in WT hyphae, compared to $\Delta a r f-b a r$ strain where no such internalization observed even after 10-15 min of FM4-64 incubation (Fig 6A). To validate these findings, the mean fluorescence intensity area was determined in biological replicates (details in subsection 'quantification and statistical analysis' of Materials and Methods). The quantitative analysis of FM4-64 data suggested that the endocytic activity from the plasma membrane of $A$. rabiei cells was reduced by $49.31 \%$ in $\Delta$ arf-bar mutant strain $(\mathrm{n}=12, p=0.0002$; Fig $6 \mathrm{~A})$. The result provides evidence that $A r F-B A R$ regulates endocytosis in A. rabiei.

Central components of the endocytic pathway are the EEs, where the small GTPase Rab5 plays a major regulatory role in their biogenesis [37]. ArRab5, an orthologue of Rab5, was identified in the A. rabiei genome using Rab5 of M. oryzae and U. maydis as query at NCBI. The relationship between ArF-BAR-associated endocytosis and ArRab5-associated early endosomes was determined using a double-labeling experiment. The T-DNA constructs having $A r F-B A R$ tagged with $m$ Cherry and ArRab5 tagged with EGFP were sequentially transformated into the WT A. rabiei strain. The coalescence of fluorescence showed perfect positive correlation, for the co-localization, between ArRab5 and ArF-BAR chimeric proteins ( $\mathrm{n}=22$; Fig 6B).

Early endosomes mature to late endosomes followed by the replacement of Rab5 by Rab7 [38]. Thus, we aimed to determine whether the punctate distribution of ArF-BAR was associated with all endocytic vesicles or specifically to the EEs. In this context, similar to Rab5 orthologue, an orthologue of Rab7 was identified in A. rabiei and was tagged with EYFP (ArRab7: $E Y F P)$. A similar double-labeling experiment was performed that showed separate intensity and localization of ArF-BAR::mCherry and ArRab7::EYFP chimeric proteins (Fig 6C). In summary, these results show association of ArF-BAR with early endocytic vesicle protein complexes and defect in FM4-64 endocytosis suggest a role in endocytosis.

\section{ArF-BAR modulates the actin cytoskeleton}

The presence of SH3 (SRC homology 3) domains in F-BAR proteins is well documented for their relationship with the actin cytoskeleton via interactions with the Arp2/3 complex activator Wiskott-Aldrich syndrome protein (WASp) [39]. Since, ArF-BAR in A. rabiei, contains two consecutive SH3 domain at its C-terminus, we initially hypothesized the existence of interactions of ArF-BAR with ArActin. Using Y2H system, it was shown that ArF-BAR protein does not interact with actin (Fig 7A), rather it physically interacts with WASp through its SH3 domains (Amino acids 572-760; Figs 7B and S12A and S12B). Further, to check the role of ArF-BAR in actin polymerization, a well-established in vitro actin polymerization assay was performed. The kinetics of actin polymerization was monitored by increase in the fluorescence of pyrene-labeled actin. The effect of purified ArF-BAR protein on actin nucleation (actin, Arp2/3 and WASp) was tested using a minimal set of components for all reactions. Interestingly, the addition of purified recombinant protein led to an increase in the actin polymerization rate (Fig $7 \mathrm{C})$. By increasing the concentration of purified protein, a significant gradual activation in the rate of actin polymerization was observed (Fig 7C). These results strongly suggest that ArF-BAR plays an active role in WASp-dependent actin polymerization. 
A

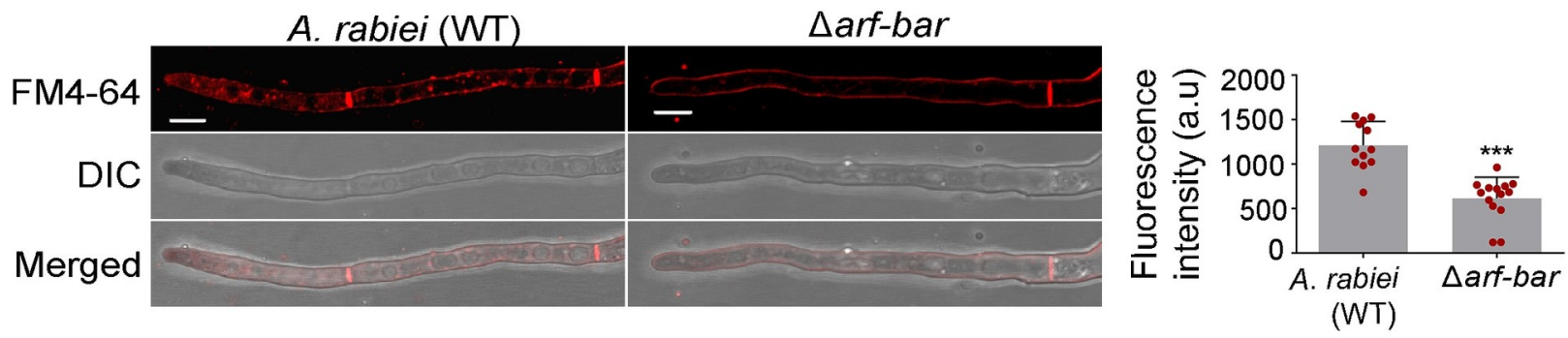

B

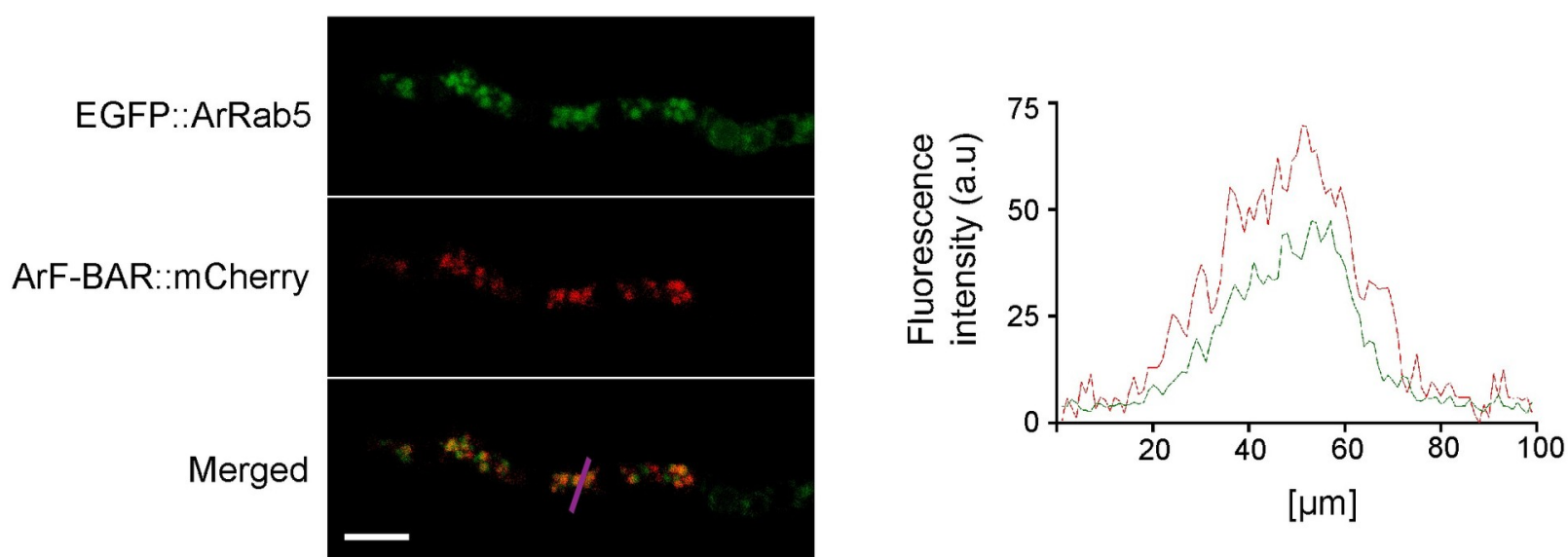

C

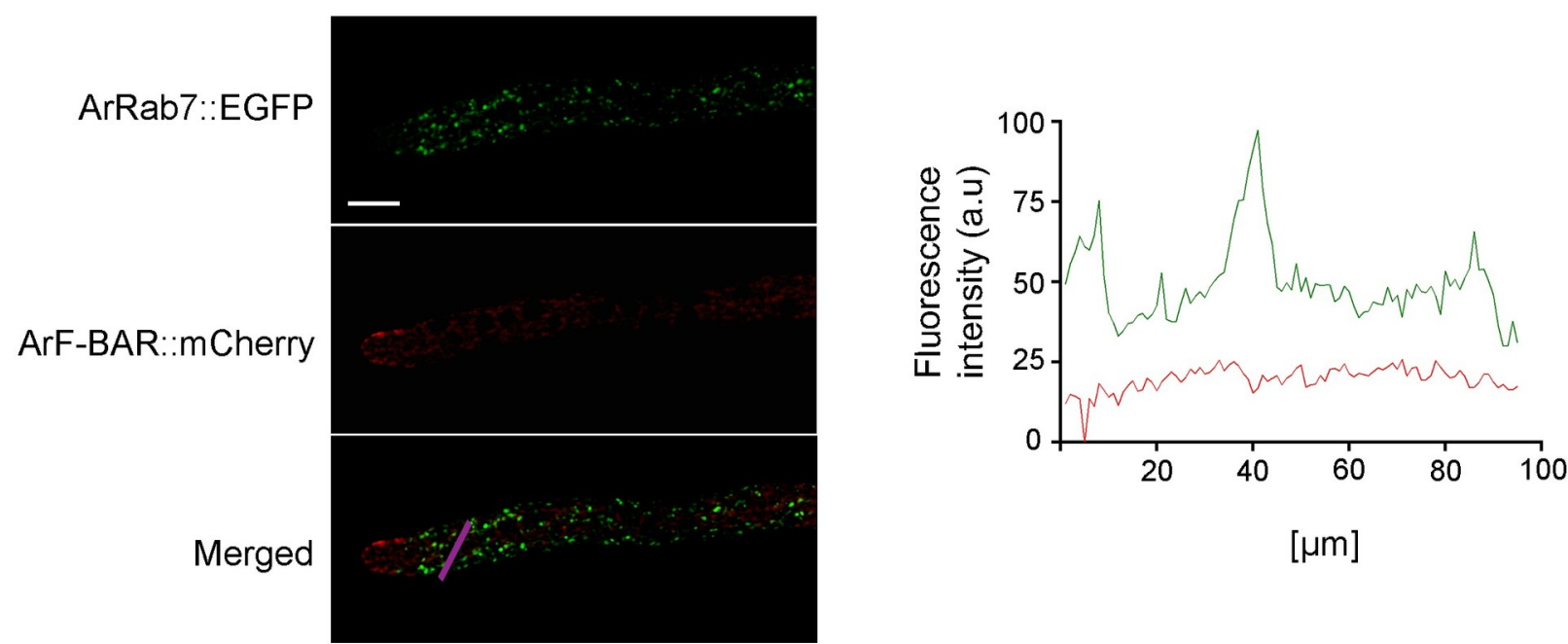

Fig 6. ArF-BAR facilitate endocytosis. (A) Confocal images of A. rabiei (WT) and $\Delta a r f-b a r$ hyphae after 10 min incubation with FM4-64 to acquire internalization capacity. Scale bar $=5 \mu \mathrm{m}$. Right panel represents the bar graph of FM4-64 fluorescence intensity per unit area mean value (a. $\mathrm{u}=$ arbitrary unit) in WT A. rabiei $(1210.78 \pm 256.18)$ and $\Delta$ arf-bar mutant $(604.092 \pm 246.62)$ strains hyphae after internalization of the dye. The significance of mean fluorescence intensities variation was statistically examined using one-tailed paired t-test, ${ }^{* * *} p=0.0002$. Red dots represent the value of each replicate. (B) Confocal images showing co-localization of ArF-BAR::mCherry with EGFP::ArRab5 ( $\mathrm{n}=22)$, scale bar $=10 \mu \mathrm{m}$. Right panel shows the positive correlation between the fluorescence intensity of ArF-BAR (Red) with ArRAB5 (Green) across the magenta line in merged panel. (C) ArF-BAR does not localizes with the late endosome marker ArRab7::EYFP. The representative image is the maximum intensity projection of all $z$-stack 
images with $1 \mu \mathrm{m}$ step size. Images were acquired after $12 \mathrm{~h}$ post-germination of fungal conidia expressing ArF-BAR::mCherry and ArRab7::EYFP. Scale bar $=5 \mu \mathrm{m}$. The images were acquired from five biological replicates with each having five technical replicates.

https://doi.org/10.1371/journal.pgen.1009137.g006

Appropriate organization of actin is required for vesicular dynamics, organelle movement and cytokinesis. Actin microfilaments or F-actin are organized into higher order structures comprising of actin patches, cables and rings that serve as the track for long distance transport [40]. To elucidate the relative importance of ArF-BAR in actin organization, actin dynamics was compared in the WT and $\Delta a r f-b a r$ mutants. Here, we took advantage of Lifeact, an actin binding peptide fused with a fluorescence protein. Lifeact has been successfully employed for in vivo visualization of actin filaments and dynamics in a variety of organisms including fungi and plants [41]. In this study, a Lifeact:mCherry fusion construct was generated and transformed into both the WT and $\Delta a r f-b a r$ mutant to visualize cytoplasmic actin dynamics in fungal hyphae. Confocal microscopy revealed discrete actin patches and cables in the WT hyphae, while actin patches were rarely visible in the $\Delta$ arf-bar mutant (WT $=92 \pm 19.86$ and $\Delta a r f-b a r=$ $16.3 \pm 4.49 ; p=0.0272$; Fig $7 \mathrm{D}$ and 7E). Moreover, actin cables were dramatically disorganized in the $\Delta a r f-b a r$ mutant. Although ArF-BAR does not directly interact with actin, it regulates actin polymerization and actin cytoskeleton through its association with WASp in growing fungal hyphae.

\section{ArCRZ1 is a potential transcriptional regulator of $A r F-B A R$ expression}

Thus far, the findings of the present study have revealed the importance of ArF-BAR in the regulation of endocytic pathways, which is crucial for the pathogenesis of A. rabiei. However, the transcriptional regulators associated with the expression of endocytic pathway genes in filamentous pathogenic fungi are largely unknown. Hence, the transcriptional mechanism associated with the regulation of $A r F-B A R$ expression during host infection was analyzed. The known core-binding motifs for fungal TFs were identified in the 727 bp $5^{\prime}$ upstream regulatory genomic sequence of $A r F-B A R$ gene by YEASTRACT online tool [42]. It revealed seven different putative TF binding sites in the $5^{\prime}$ upstream regulatory region between $A r F-B A R$ and ST47_g8004 genes (S1 Table). Among these, three core-binding sites (GAGCCC) were found for calcineurin-responsive zinc finger transcription factor 1 (CRZ1), positioned at $-106,-136$, and -254 upstream to the ArF-BAR translational start site ATG (Fig 8A).

CRZ1 is an evolutionarily conserved TF from yeast to mammals. CRZ1 was chosen for analysis because it regulates the expression of various genes involved in stress tolerance [43] and is widely known to translocate inside the nucleus with an increase in cytosolic $\mathrm{Ca}^{2+}$ ion concentration [44]. Ascochyta rabiei gene (ArCRZ1; ST47_g3738) encodes a protein that possesses a serine-rich region (SRR), two consecutive calcineurin docking domains (CDD), characterized by PxIxIT motif (PRILPQ and PEINID) and a single $\mathrm{C}_{2} \mathrm{H}_{2}$ zinc finger motif (S13A Fig). To determine the role of ArCRZ1 in the transcriptional regulation of $A r F-B A R$, the binding of ArCRZ1 to the regulatory sequences of $A r F-B A R$ was confirmed. This confirmation was performed via an electrophoretic mobility shift assay (EMSA) using recombinant His-tagged ArCRZ1 proteins. Shifting was observed for the DNA fragment possessing the calcineurindependent response element (CDRE) in the presence of purified His-ArCRZ1. However, mutations in this CDRE resulted in the abolishment of binding (Fig 8B and 8C).

Subsequently, the sub-cellular localization of ArCRZ1 under $\mathrm{Ca}^{2+}$ and oxidative stress conditions was determined. The A. rabiei wild-type (WT) was transformed with a translational fusion of $A r C R Z 1$ with EYFP towards C-terminus. After, $0.2 \mathrm{M} \mathrm{CaCl}_{2}$ treatment, confocal microscopy revealed nuclear localization of the ArCRZ1::EYFP signal (Fig 8D). Interestingly, in absence of any treatment, the ArCRZ1::EYFP fusion protein was uniformly distributed 
A

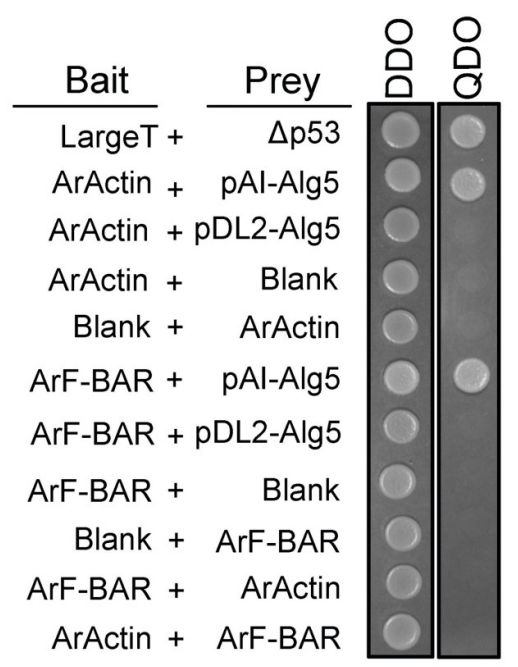

B

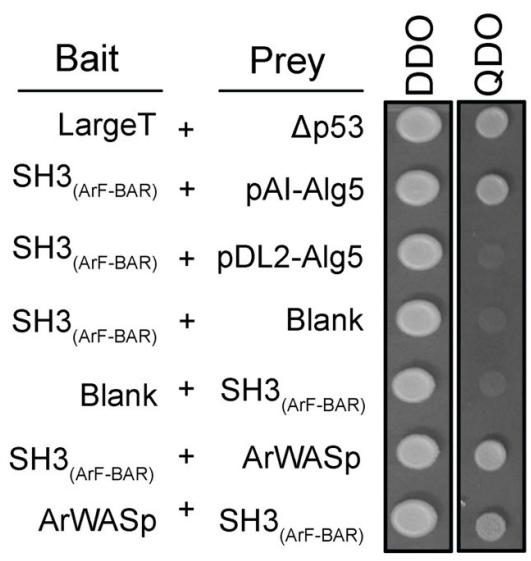

C

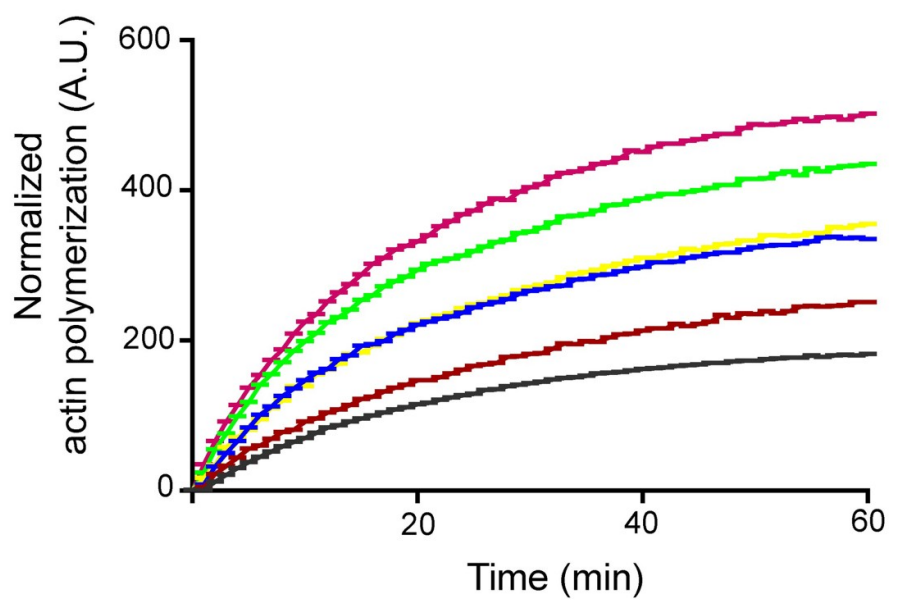

D

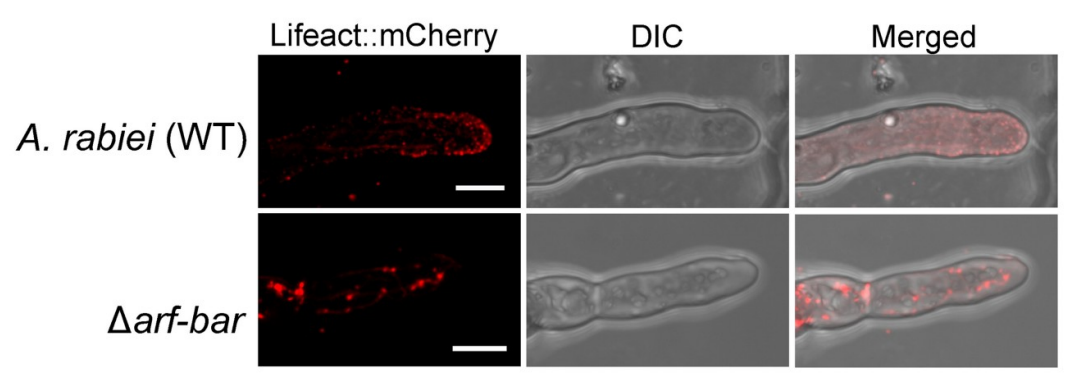

- Actin alone

- Arp2/3

- Arp $2 / 3+$ WASp

Blue + 200 nM ArF-BAR

Blue $+400 \mathrm{nM}$ ArF-BAR

Blue + 600 nM ArF-BAR

E

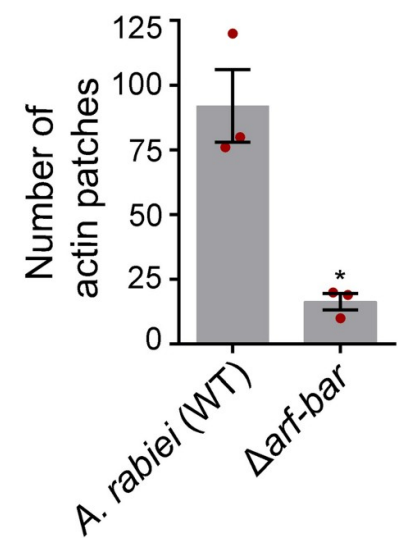

Fig 7. ArF-BAR modulates the actin cytoskeleton. (A) Split-ubiquitin based yeast two-hybrid assay to check protein-protein interaction in yeast cytoplasm. The lack of growth for yeast colonies on interaction selective QDO (SD/-L-W-A-H) media as compared to vector selective DDO (SD/$\mathrm{L}-\mathrm{W})$ suggest no interaction between ArF-BAR and ArActin. The LargeT and $\Delta \mathrm{p} 53$ are interaction positive controls while pAI-Alg5 and pDL2-Alg5 are the controls to check bait expression and autoactivation, respectively. (B) ArF-BAR exhibits positive interaction with ArWASp through its SH3 domains [SH3 (ArF-BAR); 508-760 amino acids]. The representative images were photographed $48 \mathrm{~h}$ after spotting $10 \mu \mathrm{l}$ yeast cells 
suspension. The results were evaluated with three independent biological replicates. (C) Kinetics of WASp and Arp2/3 mediated actin polymerization in absence and presence of ArF-BAR. The kinetics was measured with the change in fluorescence of pyrene-actin. For all in vitro actin polymerization assays; $4 \mu \mathrm{M}$ pyrene labelled actin, $13 \mathrm{nM}$ Arp2/3 and $15 \mathrm{nM}$ WASp were used. All the experiments were performed in three replicates. (D) Fluorescence image of Lifeact::mCherry expressed in fungal hyphae WT and $\Delta a r f-b a r$, where discrete actin patches and cables are visible in WT however actin patches are sparsely visible in $\Delta$ arf-bar. Scale bar $=5 \mu \mathrm{m}(\mathrm{n}=10)$. (E) Number of actin patches in hyphae of WT $(92 \pm 19.86)$ and $\Delta a r f-b a r(16.3 \pm 4.49)$ fungal strains, visualized by Lifeact::mCherry fluorescence. Red dots represent the mean numerical value of actin patches in three biological replicates, each having $\geq 8$ technical replicates.

https://doi.org/10.1371/journal.pgen.1009137.g007

within the cytoplasm during normal growth on glass slides (Fig 8D). Since, the nuclear translocation of CRZ1 is mediated by a phosphatase (calcineurin) [45], a chemical genetics approach was used to confirm the relationship between calcineurin and ArCRZ1. A potent calcineurin inhibitor, FK506 (Tacrolimus) was used to inhibit the enzymatic activity of calcineurin [43]. FK506 potentially inhibited nuclear translocation of ArCRZ1::EYFP, which suggests that calcineurin plays a role in the nuclear translocation of ArCRZ1 under stress conditions (Fig 8D). To corroborate and extend these findings during infection, susceptible plants were challenged with fungal conidia expressing ArCRZ1::EYFP and nuclear localization of the chimeric protein was observed (Fig $8 \mathrm{E}$ ). Overall, these results confirm the evolutionarily conserved regulation of ArCRZ1 by calcineurin under stress conditions.

To uncover the transcriptional regulation of the ArF-BAR gene mediated by ArCRZ1, a targeted deletion mutant strain of the $A r C R Z 1$ gene was generated ( $\triangle \operatorname{arcrz1}$; S13B), followed by complementation of mutant with $A r C R Z 1$ controlled by native promoter ( $\triangle \operatorname{arcrz1/ArCRZ1;}$ S13C and S13D Fig). Interestingly, no significant difference in radial growth diameter was observed between $\triangle a r c r z 1$ mutant and A. rabiei wild-type (WT) (Fig 9A). The expression pattern of $A r F-B A R$ in the WT and $\triangle a r c r z 1$ was analyzed using semi-quantitative RT-PCR. The results clearly showed significant reduction in ArF-BAR transcription in the $\triangle a r c r z 1$ mutant (Fig 9B). Imposing oxidative stress to both the WT and the $\triangle$ arcrzl mutant via menadione treatment revealed an upregulation of the $A r F-B A R$ transcript in the wild-type. However, this upregulation was found to be completely abolished in the $\Delta \operatorname{arcrz1}$ mutant (Fig 9B). Overall, these results substantiate that under oxidative stress conditions, the transcriptional regulation of $A r F-B A R$ gene is mediated by the activated ArCRZ1.

\section{Loss-of-function of $A r C R Z 1$ compromises virulence similar to $\triangle a r f-b a r$}

As ArF-BAR gene was transcriptionally regulated by ArCRZ1, it was hypothesized that the pathogenicity phenotypes of $\Delta a r c r z 1$ should be similar to those of the $\Delta a r f-b a r$ mutants. Consistent with this hypothesis, it was observed that $\Delta a r c r z 1$ mutants displayed compromised virulence during chickpea infection bioassay. Results showed that the number of lesions per plant $(\mathrm{WT}=15.31 \pm 1.44$ and $\Delta \operatorname{arcrz} 1=2.32 \pm 0.80 ; p=0.0008)$ and size of lesions $(\mathrm{WT}=4.04$ $\mathrm{mm} \pm 0.41$ and $\Delta a r c r z 1=2.03 \mathrm{~mm} \pm 0.33 ; p=0.0044)$ were lower in the $\Delta a r c r z 1$ mutant than in the wild-type $A$. rabiei. The data was obtained using $\geq 60$ chickpea plants inoculated with each fungal strain in three biological replicates. This virulence defect was rescued in $\Delta a r c r z 1 /$ ArCRZ1 complemented strain (Fig 9C-9E). The radial growth patterns of $\triangle a r c r z 1$ mutants grown on PDA supplemented with $\mathrm{Ca}^{2+}$, menadione and sodium dodecyl sulfate (SDS) were also monitored. Under these tested stress conditions, the growth inhibition was significantly greater for $\triangle a r c r z 1$ than for A. rabiei wild-type (WT). However, the growth phenotype was restored in the $\triangle \operatorname{arcrz} 1 / A r C R Z 1$ strain, which suggests that $A r C R Z 1$ plays a crucial role in calcium ion signaling, oxidative stress response and maintaining cell-wall integrity during infection (Fig 9F and 9G). Taken together, infection assay and stress treatments demonstrate that ArCRZ1 is a key regulator of $A r F-B A R$, which further regulates various cellular processes like endocytosis, effector secretion, and promotes fungal survival under host generated stress 
A

B

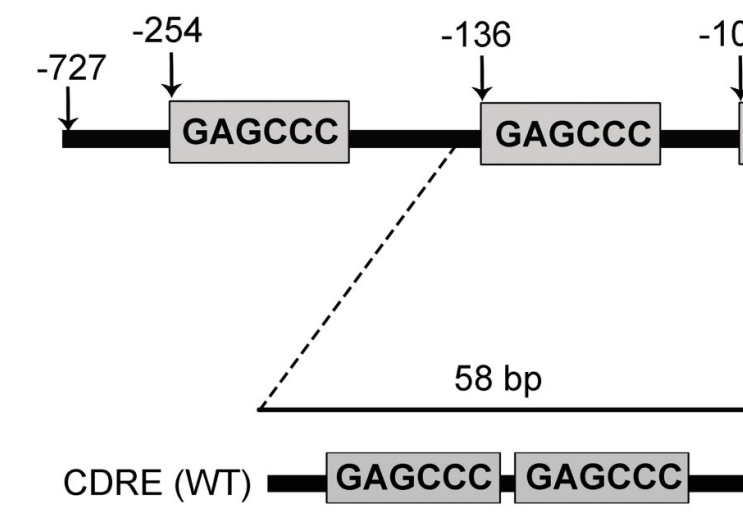

CDRE mutated (M1)

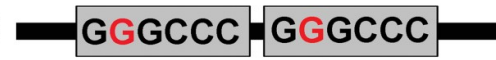

CDRE mutated (M2)

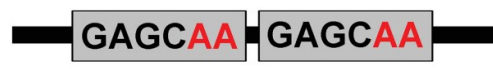

CDRE mutated (M3)

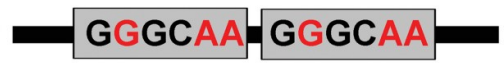

C

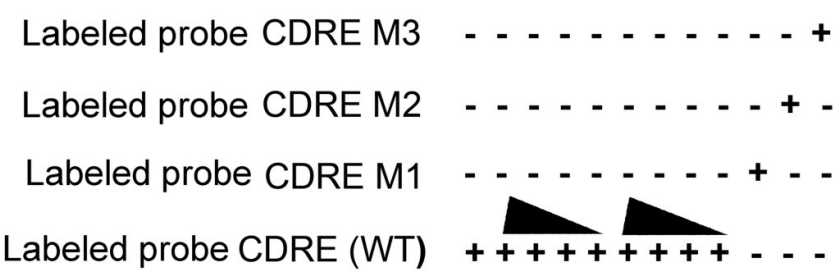

His-CRZ1

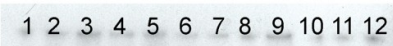

Shift $\longrightarrow$

Free probe

E

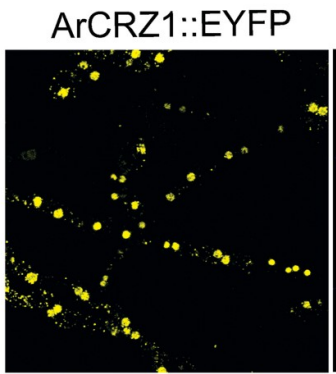

DAPI

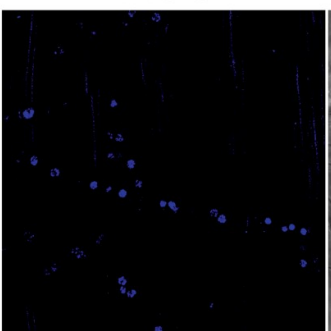

106

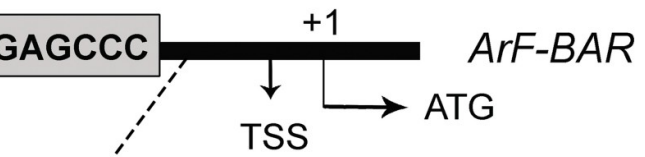

D

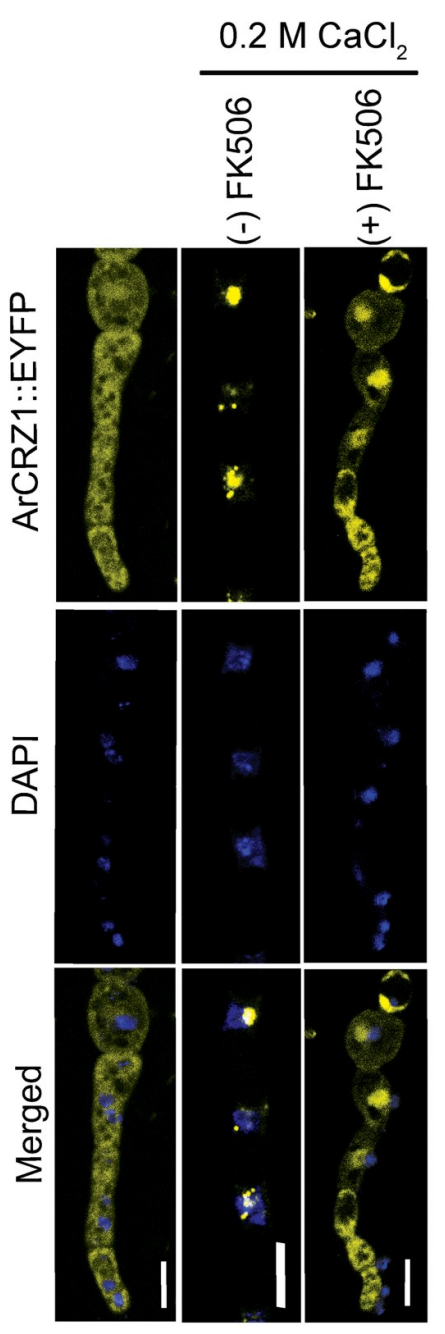


Fig 8. ArCRZ1 binds ArF-BAR promoter and localizes to nucleus under stress conditions. (A) Schematic representation of ArCRZ1 TF binding sites at the $5^{\prime}$ regulatory region of $A r F-B A R$ gene, identified by YEASTRACT online tool in 727 bp sequence. (B) Schematic representation of $58 \mathrm{bp}$ WT and mutated Calcium Dependent Response Element (CDRE) probes used for electrophoretic mobility shift assay (EMSA) of recombinant ArCRZ1. Mutated nucleotides are depicted in red colour. (C) The EMSA blot of recombinant ArCRZ1 with WT or mutated CDRE dsDNA probes. His-purified ArCRZ1 recombinant protein in 500, 600 and 200 ng quantities were used in lane 2, 3 and 4, respectively. The same protein in 800, 200 and $100 \mathrm{ng}$ amounts were used in lane 5, 6 and 7, respectively. The lane 1 and 8 contains only the dsDNA probe with protein. The plus (+) and minus (-) sign representation for labelled probes show the presence and absence of dsDNA probes. The lane 9 has WT CDRE, and lane 10,11 and 12 have mutated CDRE. This EMSA experiment was performed in triplicate. (D) Confocal images showing the cytosolic distribution of ArCRZ1::EYFP in the absence of stress condition and nuclear localization of ArCRZ1::EYFP in hyphae under stress condition $\left(0.2 \mathrm{M} \mathrm{CaCl}_{2}\right)$. Overnight grown fungal hyphae was exposed to $0.2 \mathrm{M} \mathrm{CaCl}_{2}$ two minutes prior to the microscopy. DAPI fluorescence was simultaneously recorded. Right panel in the confocal images shows the cytosolic distribution of ArCRZ1::EYFP under stress condition $\left(200 \mathrm{mM} \mathrm{CaCl}_{2}\right)$ in the presence of FK506 $(5 \mu \mathrm{g} / \mu \mathrm{l})$ calcineurin inhibition. Fungal hyphae were exposed to FK506 for $5 \mathrm{~min}$, prior to microscopy. Scale bar $=5 \mu \mathrm{m}$. (E) Confocal images showing the nuclear localization of ArCRZ1, during in planta fungal growth. Confocal images were acquired $48 \mathrm{hpi}$ of AB susceptible chickpea stem peel, infected with conidia of fungal strain expressing ArCRZ1::EYFP. Scale bar $=5 \mu \mathrm{m}(\mathrm{n}=30)$.

https://doi.org/10.1371/journal.pgen.1009137.g008

condition. Thus, ArCRZ1 induces the expression of ArF-BAR by directly binding to its cis-regulatory region and is critical for $A r F-B A R$ dependent virulence.

\section{Discussion}

Dynamic membrane remodeling is essential for maintaining the integrity and identity of cells and cellular compartments [40]. Biological macromolecules, such as BAR superfamily proteins, can sense or induce membrane curvature. They are well-known modulators of transient membrane deformation [39]. Emerging evidence strongly suggests that EEs are crucial for long-distance intracellular communication. Thus, EEs and their roles have broad implications for a wealth of cellular processes such as growth, development, and virulence in filamentous fungi $[4,46,13]$. The results of the present study further corroborate this conclusion and provide new information regarding signaling and transcriptional control of F-BAR proteins in phytopathogenic fungi. To date, our understanding of the role played by F-BAR proteins in membrane curvature generation and efficient long-range endosome trafficking in fungi during plant-pathogen interactions remains very limited.

To best of our knowledge, this study provides the evidence that an F-BAR domain protein acts as a key mandate for virulence of a phytopathogenic fungi. Based on the evidences provided in the present study, we propose that ArF-BAR contributes to endocytosis, effector secretion, septa formation, and actin dynamics during the A. rabiei hyphal growth (Fig 10).

In a first set of evidences, our results indicate the role of membrane remodeling in septa formation and hyphal tip growth. Previous studies, largely conducted in animal models, have highlighted that the F-BAR domain is a membrane-deforming module and is involved in endocytosis $[47,29]$. The endocytic event is crucial for the uptake of signal cues and nutrition from the host, and aids apical recycling of membrane receptors and proteins. This process thus helps to maintain the overall polarity of the hyphae that is required for fungal growth and virulence $[6,43]$. The generation of EEs and their trafficking involves microtubule dynamics, actin cytoskeleton rearrangements and most importantly, extensive membrane remodeling [48]. Actin dynamics and microtubule organization have been extensively studied. However, the detailed mechanism underlying the functional regulation of fungal EE biogenesis and EE trafficking at the hyphal tip during plant pathogenesis remains poorly understood.

The present study identifies the role for the ArF-BAR protein as an essential component of endocytosis. ArF-BAR in coordination with Arp2/3-WASp, was found to mediate actin cytoskeleton assembly at the hyphal tip of growing end. The Arp $2 / 3$-WASp assembly is a prerequisite for host penetration [33,49]. The early endosomes move on molecular tracks of F-actin network [8,39], which was disorganized in the $\Delta a r f-b a r$ mutant, affecting endocytic transport. 
A

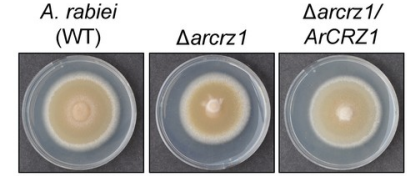

$\mathbf{F}$

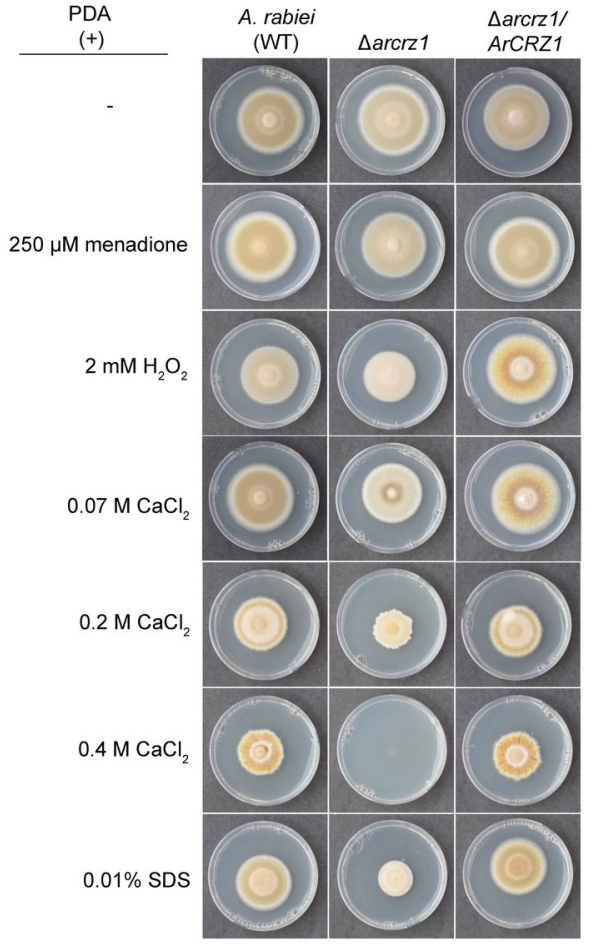

B

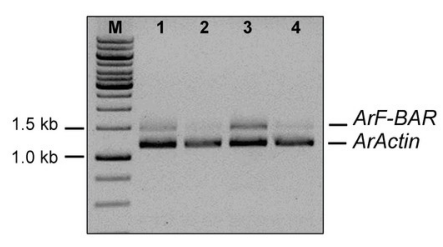

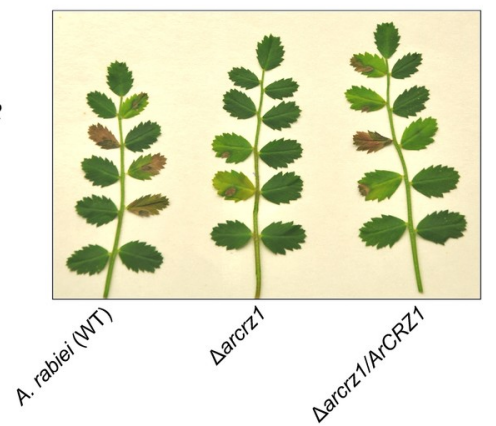

E

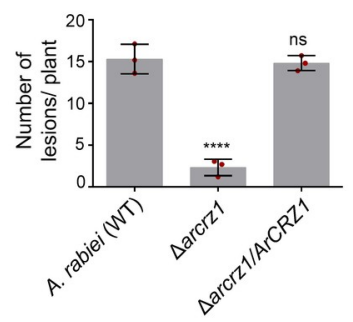

G

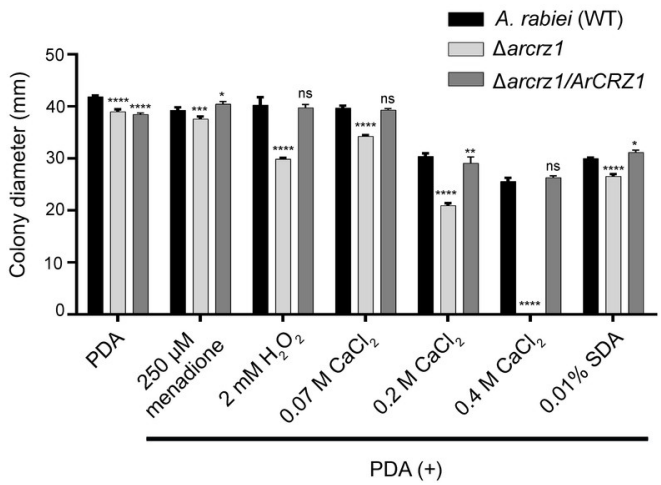

Fig 9. ArCRZ1 regulates ArF-BAR transcription and A. rabiei virulence. (A) Vegetative growth phenotype of 7 days old A. rabiei (WT), $\triangle a r c r z 1$ and $\triangle a r c r z 1 / A r C R Z 1$ strains grown on PDA plate. (B) Expression analysis of $A r F-B A R$ gene in $\triangle a r c r z 1$ through semi-quantitative PCR. Lane 1 and 2 shows $A r F-B A R$ expression in mycelia of $A$. rabiei (WT) and $\triangle \operatorname{arcrz} 1$ while lane 3 and 4 shows $A r F-B A R$ expression in $A$. rabiei (WT) and $\triangle a r c r z 1$ after $0.5 \mathrm{~h}$ treatment with $250 \mu \mathrm{M}$ menadione to the axenic culture of mycelia in PDB. (C) Representative image of disease symptoms obtained on Ascochyta blight susceptible chickpea 6 days post inoculation (dpi) of A. rabiei (WT), $\Delta a r c r z 1$, and $\Delta a r c r z 1 /$ ArCRZ1 strains. (D) Statistical analysis of the AB disease symptoms on chickpea plants inoculated with three different $A$. rabiei strains. The height of bar in graph shows the mean value of lesions per plant $(\mathrm{WT}=15.31 \pm 1.44, \triangle \operatorname{arcrz} 1=2.32 \pm 0.80$, and $\triangle \operatorname{arcrz} 1 / \operatorname{ArCRZ1}=$ $14.82 \pm 0.73)$. (E) The bar height in graph represents the mean value of lesion size, in diameter (WT $=4.04 \mathrm{~mm} \pm 0.41, \Delta \operatorname{arcrzl}=2.03$ $\mathrm{mm} \pm 0.33$, and $\triangle \operatorname{arcrz} 1 / \operatorname{ArCRZ1}=3.98 \mathrm{~mm} \pm 0.56$ ). The mean and standard deviation ( \pm ) were calculated from three biological replicates, counting at least 20 plants for each replicate. These results were quantified using one-way ANOVA, compared with the control $\left({ }^{* * * *} p<0.0001\right)$. Each red dot represents the mean value of biological replicate. (F) Colony morphology and growth assay of WT A. rabiei, $\triangle a r c r z 1$ mutant and complemented $\triangle \operatorname{arcrz} 1 / \operatorname{ArCRZ1}$ fungal strains observed under various stress conditions after 10 days incubation at $22^{\circ} \mathrm{C}$. PDA medium was separately supplemented with $250 \mu \mathrm{M}$ menadione, $2 \mathrm{mM} \mathrm{H}_{2} \mathrm{O}_{2}, 0.07 \mathrm{M}, 0.2 \mathrm{M}, 0.4 \mathrm{M} \mathrm{CaCl}_{2}$ and $0.01 \%$ SDS. The $\Delta$ arcrz1 mutant exhibited enhanced sensitivity towards stress conditions as compared to A. rabiei (WT). The mutant strain was highly sensitive towards $\mathrm{CaCl}_{2}$ and failed to grow at $0.4 \mathrm{M} \mathrm{CaCl}_{2}$. (G) The bar graph represents diameter values (mean and SD) of the radial growth for three fungal strains. All the growth assays were performed in triplicates. The results were quantified using two-way ANOVA, Tukey's multiple comparisons. The statically significant differences are shown with ${ }^{* * * *} p<0.0001,{ }^{* * *} p<0.001,{ }^{* *} p<0.01,{ }^{*} p<0.05$, ns $=$ non-significant.

https://doi.org/10.1371/journal.pgen.1009137.g009

The loss-of-function mutant of the $A r F-B A R$ gene leads to the substantial attenuation of virulence compared to WT. This loss in virulence is similar to that observed in the rice blast fungus $M$. oryzae and $U$. maydis where endocytosis is crucial for the recognition of host partners 


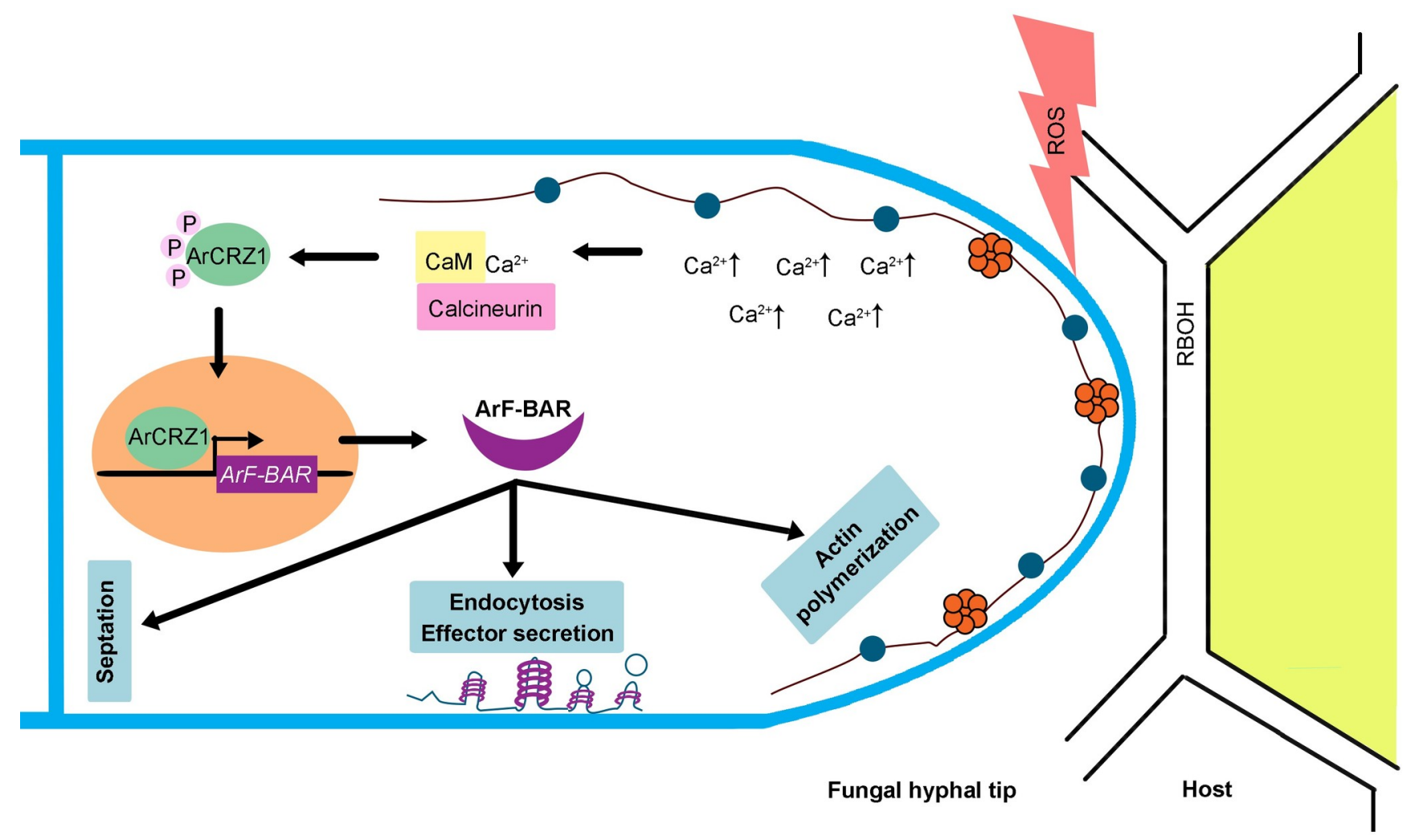

Fig 10. ArF-BAR regulated functions in A. rabiei cells and its regulation by ArCRZ1. On encounter with the pathogen, plant generates various stresses around the site of pathogen recognition; a common stress is oxidative stress. The perception of these stresses by the pathogen, results in the upregulation of cytosolic $\mathrm{Ca}^{2+}$ within the pathogen. Here, in A. rabiei, increased calcium level, sensed by calmodulin mediates the activation of calcineurin. Activated calcineurin dephosphorylates cytoplasmic inactive ArCRZ1 (phosphorylated form of ArCRZ1 remains inactive). Dephosphorylation of ArCRZ1 results in its translocation to the nucleus where it regulates the expression of $A r F-B A R$ and other genes to cope with stress. Further, during polarized growth this ArF-BAR gets localized to hyphal tip where it leads to generation and stabilization of membrane curvature which is crucial for endocytosis and helps in the secretion of effector proteins required for fungal virulence. Additionally, ArF-BAR protein mediates actin organization and helps in septa formation.

https://doi.org/10.1371/journal.pgen.1009137.g010

during the early stages of pathogenic development [7]. However, the slow growth of the mutant (Figs 3 and S8) may be the possible cause of the reduced virulence by itself. We observed that the symptoms in plant bioassay (Figs 2 and S6) are severe in case of WT. The $\Delta a r f$-bar mutant showed only small or almost no lesions on plants. These observations clearly indicated that such severity is not merely due to slow growth of hyphae. Therefore, we hypothesized that the knockout mutant may have impaired in ability to secrete effectors of $A$. rabiei. Fungal pathogens secrete an array of effector proteins to counter host immunity and promote infection process [50]. $\Delta a r f$-bar mutant strain was found defective in secretion of a secretary signal sequence containing effector, Ar93 (Fig 5D). This experiment clearly shows that cumulative effect of both the slow growth and effector secretion are the primary cause of severe virulence defects in $\Delta a r f$-bar mutant. Additionally, some of the fungal effectors can change the structural and functional conformations as well as the localization of host target proteins that are key factors in host ROS production [51]. Pathogen survival under host-generated ROS is important for fungal growth and virulence. We also observed high transcript accumulation of ArF-BAR under oxidative stress conditions (Fig 1B). However, it will be interesting to decipher the entire mechanism of pathogen survival correlating all the components together.

As discussed above, during host-pathogen interactions, the key to successful pathogenesis is to overcome the rigid defense responses of the host. Collectively, the first challenge the 
pathogen encounters is an oxidative burst at the site of infection, which initiates various signaling cascades in the pathogen that aid its survival. Calcium, an essential secondary messenger, mediates one such signaling cascade [52]. In response to stimuli, the cytosolic $\mathrm{Ca}^{2+}$ concentration increases [53] and modulates various $\mathrm{Ca}^{2+}$-binding proteins such as calmodulin. The $\mathrm{Ca}^{2}$ ${ }^{+}$and calmodulin complex activates calcineurin. In many eukaryotes, calcineurin is known to regulate the activity of CRZ1, which is usually localized in the cytosol in phosphorylated form. Upon activation, CRZ1 gets relocated to the nucleus. In pathogenic fungi, many of the CRZ1-dependent targets, such as those involved in the maintenance of cell wall integrity, thermo-tolerance, cation homeostasis, azole tolerance, and hyphal growth have been identified [54]. Here, our study assumes functional significance for plant infection as we identified an F-BAR protein that is regulated by transcriptional network. We have identified the calciumregulated CRZ1 transcription factor that regulates the transcription of F-BAR. These results, improves our understanding of the novel regulatory mechanism of endocytosis in filamentous fungi, where ArF-BAR functions downstream of ArCRZ1.

The development of septa is an important event during hyphal differentiation that is required for the formation of sexual structures and asexual spores [53]. Septation is comparable to cytokinesis that additionally includes cell separation. A cascade of events is involved in septum biogenesis, which includes assembly of the contractile actomyosin ring (CAR), plasma membrane ingression and cell wall constriction [55]. In fission yeast, Cdc15 and Imp2, and in budding yeast, Hof1 are the major F-BAR domain-containing proteins implicated in the formation of the CAR, and the primary and secondary septa during cytokinesis [56,57]. Additionally, U. maydis Cdc15 is also implicated in septin ring formation [16]. Therefore, the localization of ArF-BAR along the septum ring indicates that endocytosis is one of the pathways responsible for regulating the development of the septa. Septation is initiated at the definitive size of the hyphae [58]. With the initial recognition and establishment of disease in the host, the pathogen needs to proliferate at an enormously increased rate. Therefore, maintaining proper hyphal architecture and polarity is fundamental for pathogenesis that demands rapid coordinated internalization and recycling events [59]. The fungal mutant $\Delta a r f-b a r$, deficient in ArF-BAR protein, revealed delay in septa formation and displayed decreased virulence in the absence of proper hyphal structure. In summary, we propose that the ArF-BAR protein of $A$. rabiei has the potential to interactively affect the hyphal growth and virulence of filamentous fungus. This evolving model provides mechanistic insight into the role of a membrane scaffolding protein in the process of endosome trafficking in fungal pathogenesis. In turn, this provides many additional potential targets for the development of effective and durable strategies to control $\mathrm{AB}$ fungal disease. Therefore, the observations of the present study in context to the intracellular trafficking, during the early stages of plant-pathogen interactions, have broad relevance for shaping disease-control strategies. These findings may also be helpful for the disease control of animal-infecting fungal pathogens such as A. fumigatus and C. albicans. Thus, further studies will be directed to characterize the interacting partners influenced by ArF-BAR during endosome formation. Further, the understanding of its regulatory mechanism would help in scrutinizing the molecular and cellular basis of disease development, which would subsequently help develop disease-control strategies for filamentous fungi.

\section{Materials and methods}

\section{Fungal strains and growth conditions}

A virulent isolate of Ascochyta rabiei (ArD2; ITCC No. 4638) was procured from IARI, New Delhi. The single spore culture of this mating type 2 isolate was generated and maintained as wild type fungi for research work. The WT and its derivative fungal strains (S2 Table) were 
maintained on potato dextrose agar (PDA; Difco Laboratories, $\mathrm{pH} 5.2-5.5$ ) at $22^{\circ} \mathrm{C}$ for $10-15$ days to assess the growth pattern and colony characteristics [26]. The fungus was routinely subcultured on PDA plates supplemented with chickpea extract to maintain virulence. To determine the vegetative growth pattern of fungal mycelia in response to oxidative stress, PDA plates supplemented with menadione $\left(250 \mu \mathrm{M}\right.$ and $500 \mu \mathrm{M}$; Sigma-Aldrich, USA) or $\mathrm{H}_{2} \mathrm{O}_{2}(2$ $\mathrm{mM}$; Sigma-Aldrich, USA). The conidial suspensions of $10 \mu \mathrm{L}\left(1 \times 10^{3}\right.$ conidia $\left./ \mathrm{mL}\right)$ were inoculated at the centre of PDA plates for growth assay. After 10 days of incubation under optimum growth conditions, the diameters of fungal colonies were measured using ImageJ software. Three independent biological experiments were performed with three technical replicates each time.

\section{RNA extraction and expression analysis}

Total RNA was extracted from 5-6 days old fungal mycelia grown in potato dextrose broth (PDB; Difco Laboratories, USA) or from plant tissues inoculated with A. rabiei wild-type (WT) using TRIzol reagent (Invitrogen, USA). The isolated total RNA was subjected to DNase1 (Promega, USA) treatment and subsequently used for first-strand cDNA synthesis using SuperScript IV reverse transcriptase (ThermoFisher Scientific). Targeted gene expression was determined by qRT-PCR with ABI7900 (Applied Biosystems, USA), using SYBR Green PCR master mix (Applied Biosystems, USA). Relative expression of fungal genes was calculated after normalization with elongation factor $\alpha\left(A r E F \alpha\right.$; ST47_g4052) using this $2^{-\Delta \Delta c t}$ method [60]. The data were analysed from three biological replicates each having three technical replicates.

\section{Site-directed mutagenesis}

The mutations at required sites of $A r F-B A R$ were achieved by PCR amplification of pET28a $(+): A r F-B A R$ clone with pre-designed primers containing mutations of interest. Mutagenesis was performed using the QuikChange II Site-directed mutagenesis kit (Agilent, USA). Webbased Quik Change Primer Design tool (www.agilent.com/genomics/qcpd) was used to design primers. The presence of mutations in clones was confirmed by Sanger sequencing.

\section{Targeted gene knockout and complementation in A. rabiei}

Homologous gene replacement with $h p h$ cassette strategy was used to generate knockout (KO) constructs for A. rabiei genes. Genomic DNA was isolated from 5-day grown PDB culture of A. rabiei using GenElute Plant Genomic DNA miniprep kit (Sigma-Aldrich, USA). The $5^{\prime}$ flanking genomic sequences of $A r F-B A R$ were amplified from $A$. rabiei genomic DNA using primer pairs of ArF-BARKOif5F and ArF-BARKOif5R while the $3^{\prime}$ flanking sequences were amplified using Ar72KO3 and Ar72KO4 (S3 Table). These amplified 5' and $3^{\prime}$ flanking sequences were cloned sequentially into pGKO2 vector at $K p n \mathrm{I} / P s t \mathrm{I}$ and $B a m \mathrm{HI} / E c o$ RI sites, respectively. The cloned $A r F-B A R$ gene replacement cassette of $\sim 3.4 \mathrm{~kb}$, including $5^{\prime}$ and $3^{\prime}$ flanking sequence along with $h p h$, was amplified using primer pair ArF-BARKOif5F and Ar72KO4, and transformed into A. rabiei protoplasts. The A. rabiei protoplast transfection was performed as described earlier [61], with some minor modifications. The putative transformants were selected on a PDA plate supplemented with $50 \mu \mathrm{g} / \mu \mathrm{L}$ hygromycin. To generate the complementation constructs of $A r F-B A R$ and its mutated (mut1 and mut2) versions, about $4.4 \mathrm{~kb}$ DNA fragment having the $A r F-B A R$ native promoter, ORF region, and TrpC terminator was amplified and cloned into pBIF2 vector (Bacterial selection- kanamycin; Fungal selectionG418) at EcoRI and HindIII sites. These three constructs of ArF-BAR were independently transformed into $\triangle a r f$-bar mutant strain by Agrobacterium tumefaciens-mediated 
transformation (ATMT) [27]. Similar to generation of ArF-BAR knockout and complementation mutant, A. rabiei ArCRZ1 gene knockout mutant and complementation strains were also developed. A. rabiei ArCRZ1 gene knockout mutant and complementation strains were developed with oligonucletides enlisted in S3 Table.

\section{Gene knockout confirmation by PCR and Southern blot}

The single spore culture of putative KOs selected on hygromycin was initially screened by genomic PCR. To check the proper recombination of deletion construct at desired genomic region, a primer set binding at position $5^{\prime}$ to the homologous recombination region and TrpC promoter was used for gene $5^{\prime}$ upstream region while a primer set binding at $H p h$ gene and position $3^{\prime}$ to the homologous recombination region was used for gene $3^{\prime}$ downstream region ( $\mathrm{S} 3 \mathrm{Table})$. The putative transformants for complementation strains were also screened by genomic PCR. The PCR positive $\Delta a r f-b a r, \Delta a r c r z 1$ mutants (KOs), and complemented KO strains were further verified by Southern blot. The genomic DNA of WT, $\Delta a r f-b a r$ and $\triangle a r c r z 1$ mutants was digested with EcoRI enzyme while genomic DNA was digested with EcoRI and HindIII for the complementation strains ( $\triangle a r f-b a r / A r F-B A R, \triangle a r f-$ bar/ArF-BAR ${ }^{\text {mut1 }}, \triangle a r f-b a r / A r F-B A R^{\text {mut2 }}$, and $\left.\triangle \operatorname{arcrz1} / A r C R Z 1\right)$. The digested DNA was separated along with $\lambda$ DNA/HindIII marker (ThermoFisher Scientific) and blotted to a membrane followed by hybridization with radioactive probe prepared using the random primers labeling NEBlot kit (New England Biolabs, USA). The band detection was carried out using Typhoon phosphor imager (GE Healthcare, USA).

\section{Plant infection assay}

Two-weeks-old susceptible chickpea (Pusa 362) plants grown in plant growth chambers under controlled conditions (D/N temperature: $24^{\circ} \mathrm{C} / 18^{\circ} \mathrm{C}$; Relative Humidity: $80 \%$; light intensity $250 \mu \mathrm{E} / \mathrm{m}^{2} / \mathrm{s} ; \mathrm{D} / \mathrm{N}$ light duration: $14 / 10 \mathrm{~h}$ ) were used for infection assays. Conidial suspensions of A. rabiei strains were collected separately from a 20-days-old PDA plate grown culture. Two-weeks-old plants were spray inoculated with conidial suspensions diluted to $2 \times 10^{6}$ conidia/mL. Plants were again kept under optimum conditions. Disease lesions were examined 5-7 days after spray inoculation.

\section{In vitro protein purification}

The ORFs of desired genes were amplified from cDNAs of the interest, cloned in pET28a $(+)$ and transformed into E. coli BL21-CodonPlus (DE3)-RIPL cells. Protein expression was induced with $0.5 \mathrm{mM}$ Isopropyl $\beta$-D-thiogalactoside (IPTG) for $6 \mathrm{~h}$ at $23^{\circ} \mathrm{C}$. Bacterial pellet was lysed in buffer [500 mM NaCl, $50 \mathrm{mM} \mathrm{NaPO}_{4}(\mathrm{pH} 8.0), 10 \mathrm{mM}$ Imidazole, $1 \mathrm{mM} \beta$-Mercaptoethanol, $1 \mathrm{mg} / \mathrm{mL}$ lysozyme and $1 \mathrm{mM}$ Phenylmethylsulfonyl fluoride (PMSF)] by incubating $30 \mathrm{~min}$ on ice, followed by sonication ( 10 cycle, $70 \%$ amplitute). The cell lysates were precipitated followed by $0.45 \mu \mathrm{m}$ filtration. The cleared lysate was incubated with Ni-NTA resin for $30 \mathrm{~min}$ at $4^{\circ} \mathrm{C}$. The His-tagged fusion proteins were purified using a Ni-NTA column (Bio-Rad, USA). Proteins were eluted in elution buffer [500 mM NaCl, $50 \mathrm{mM} \mathrm{NaPO}_{4}(\mathrm{pH}$ 8.0), $10 \%$ glycerol and $200 \mathrm{mM}$ imidazole]. The quality and quantity of eluted protein were checked by SDS/PAGE and Bradford assay, respectively. The proteins were dialyzed in compatible buffers, as per experiment, followed by concentration. 


\section{Liposome preparation and tubulation assay}

Liposomes were prepared using 70\% Phosphatidylethanolamine (POPE), 20\% Phosphatidylcholine (POPC), and 10\% Rhodamine B-conjugated PE (Echelon Biosciences, USA). In an amber glass vial, all the lipids were initially dissolved in chloroform: methanol $(65: 35 ; \mathrm{v} / \mathrm{v}) \mathrm{mix}-$ ture and vials were kept under liquid nitrogen for 10 min before being immediately subjected to vacuum desiccation/lyophilisation for $2 \mathrm{~h}$ at $60 \mathrm{mTorr}$. The lipids were hydrated with buffer [25 mM Tris- $\mathrm{HCl}(\mathrm{pH} 6.8)$ and $100 \mathrm{mM} \mathrm{NaCl}$ ] and subjected to three freeze-thaw cycles of 5 min each at $68^{\circ} \mathrm{C}$ and liquid nitrogen. Extrusion was performed at $68^{\circ} \mathrm{C}$ on a pre-heated mini extruder (Avanti Polar Lipids, USA). The prepared liposomes were diluted as desired and immediately proceeded for tubulation assay. Before use, the purified proteins were subjected to $100,000 \mathrm{~g}$ centrifugation for $20 \mathrm{~min}$ at $4^{\circ} \mathrm{C}$ to remove the aggregates. To examine tubule formation, the mixed liposome and protein samples were analysed in Lumox 24-well plate (Millipore, USA) by live-cell imaging on Axio Examiner.Z1 (Zeiss microscope).

\section{Liposome co-sedimentation assay}

The purified fusion proteins were pre-centrifuged, before the assay, at $100,000 \mathrm{~g}$ for $15 \mathrm{~min}$ to remove protein aggregates. Protein from the supernatant was mixed with freshly prepared synthetic liposomes with gentle tapping. Ultracentrifugation was performed at 100,000 $\mathrm{g}$ for 15 $\min$ at $4^{\circ} \mathrm{C}$ and the supernatant and the pellet were carefully separated. The supernatant was mixed with 1:1 loading buffer while the pellet was re-suspended in $2 x$ loading buffer. Samples were analysed on Coomassie stained SDS-PAGE.

\section{Yeast two-hybrid assays}

The interactions of various combinations between ArF-BAR, ArActin, ArWASP, and ArF-BAR domains in yeast cytoplasm were examined using the split-ubiquitin based DUALhunter system (Dualsystems Biotech). The ORFs were cloned at NotI and AscI restriction sites or by LR clonase II into pGDHB1 and pGPR3-N vectors. The cloned plasmids were co-transformed along with necessary controls into NMY51 strain using the EZ-Yeast transformation kit (MP Biomedicals, USA) and plated on SD/-L/-W plates. The plating of yeast clones on required synthetic media to check protein-protein interactions in yeast was done as described previously [22]. All the interactions were verified by three independent experiments.

\section{Effector secretion assay}

For total fungal protein extraction, fungal mycelia were grown in $\mathrm{PDB}$ at $22^{\circ} \mathrm{C}, 120 \mathrm{rpm}$ for 7 days and harvested by filtering through three layers of sterile Mira cloth (EMD Millipore Corp, Germany). Tissues were snap freezed in liquid nitrogen and stored in $-80^{\circ} \mathrm{C}$ for future use. The fungal tissue was grounded to fine powder and resuspended in Tris-glycine buffer $\mathrm{pH}$ 8.3 (3g Trizma, Sigma-Aldrich, USA and $14.4 \mathrm{~g}$ Glycine, Sigma-Aldrich, USA dissolved in $1 \mathrm{~L}$ $\mathrm{MQ}$ ). Resuspension was then centrifuged at $16 \times 1000 \mathrm{~g}$ for $40 \mathrm{~min}$ at $4^{\circ} \mathrm{C}$. The supernatant and cell lysate was collected separately. The total protein from the supernatant was separated on SDS-PAGE followed by immune blot with anti-Flag Ab (Cohesion biosciences, Singapore). For the extraction of secreted proteins, 7 days old grown mycelia in PDB was treated with $250 \mu \mathrm{M}$ menadione (Sigma-Aldrich, USA), to mimic host induced oxidative stress condition. Axenic culture filtrate (CF) was collected and filtered through three layered Mira cloth (EMD Millipore Corp, Germany) to separate fungal mycelia. Further, CF was sequentially filtered with 0.45 and $0.22 \mu \mathrm{m}$ Durapore PVDF membrane filters (Sigma-Aldrich, USA). Filtered CF was then concentrated using $3 \mathrm{kDa}$ Amicon Ultra-15 Centrifugal Filter Units (Merck, USA). 
The concentrated secreted proteins were then separated on SDS-PAGE followed by immunoblot with anti-Flag Ab.

\section{Actin polymerization assay}

The actin polymerization modulation activity of the proteins was checked using Actin Polymerization Biochem Kit (Cytoskeleton, USA), using the manufacturer's instruction. Freshly solubilized components- $13 \mathrm{nM}$ Arp2/3 protein complex and $15 \mathrm{nM}$ WASp-VCA domainGST purified (Cytoskeleton, USA) along with freshly purified ArF-BAR protein in concentrations of 200, 400, and $600 \mathrm{nM}$ were used. The reaction was carried out in an opaque 96-well plate and kept in the dark. The actin polymerization rate was recorded by monitoring the pyrene fluorescence signals using CLARIO star plate reader (BMG Labtech, Germany) with the following settings; slow kinetics, $60 \mathrm{sec}$ intervals, $\lambda_{\mathrm{ex}}=360 \pm 15 \mathrm{~nm}$ and $\lambda_{\mathrm{em}}=420 \pm 20 \mathrm{~nm}$.

\section{Electrophoretic mobility shift assay (EMSA)}

The His-tagged ArCRZ1 (His-ArCRZ1) expression construct was generated and recombinant protein was purified. The wild type and mutated CDRE DNA fragments were assembled by annealing oligonucleotide pairs in a thermal cycler by heating at $95^{\circ} \mathrm{C}$ for $5 \mathrm{~min}$ followed by cooling at RT for $15 \mathrm{~min}$. End labelling of DNA fragments was performed by $\left[\gamma^{32} \mathrm{P}\right]$ dATP (Board of Radiation \& Isotope Technologies, BRIT, India) and Polynucleotide Kinase (Thermo Fisher Scientific, USA). Additionally, for competition assays, two complementary oligonucleotides were annealed at equimolar concentration. Purified His-ArCRZ1 protein was incubated with $10 \mathrm{ng}$ of labelled DNA fragment in the presence of $1 \mathrm{mg}$ of poly-deoxy-inosinic-deoxycytidylic acid [poly (dI-dC)] and $1 \mathrm{X}$ binding buffer (15 mM HEPES (pH 7.6), $0.2 \mathrm{mM} \mathrm{MgCl}$, $35 \mathrm{mM} \mathrm{KCl}, 1 \mathrm{mM}$ DTT and $1 \%$ glycerol) in a reaction volume of $30 \mu \mathrm{L}$ for $25 \mathrm{~min}$ at room temperature. DNA loading dye was used to terminate the reaction. The competitive assays were performed using 50,100, and 200 times of specific fragments in excess. To identify the relative binding, the complexes were resolved on 6\% native PAGE, dried, and autoradiographed on X-ray films.

\section{Microscopic methods}

Sample preparation for microscopic analysis. Conidia from the fungal strains were harvested in $1 \mathrm{~mL}$ sterile distilled water from 15-day-old fungal mycelia grown on PDA plates. The conidial suspension was filtered through Mira cloth. Ten microlitres $\left(1 \times 10^{6}\right.$ conidia/mL) of suspension was kept on a sterile glass coverslip and allowed to grow under the optimal conditions for $24 \mathrm{~h}$ in dark. To investigate the localization of chimeric proteins in fungal hyphae, growing on chickpea stem peel, the conidia were allowed to grow for $36 \mathrm{~h}$ under optimum conditions of infection. The grown hyphae samples were then used for confocal laser scanning microscopy.

Confocal laser scanning microscopy. For microscopic studies, TCS SP5 and TCS SP8 confocal laser scanning microscopes (Leica Microsystems, Germany) were used. For subcellular localization, conidia were harvested from transgenic fungal strains expressing fluorescenttagged proteins. The Z-stacked images with $1 \mu \mathrm{m}$ step size were acquired using a high-resolution CCD camera. For calcofluor-white (CW), the hyphae grown on the glass slide were incubated for $10 \mathrm{~min}$ in the CW solution (Sigma-Aldrich, USA). After incubation, the stained hyphae were rinsed with PBS ( $\mathrm{pH}$ 7.4) followed by rinsing with sterile distilled water, before image acquisition. For FM4-64 uptake, $1 \mathrm{~mL}$ of harvested conidial suspension was centrifuged at 2,500 g, washed twice with sterile distilled water and then allowed to germinate on glass slides. The aqueous solution $(10 \mu \mathrm{M})$ of FM4-64 dye (Invitrogen, USA) was added directly to 
the fungal mycelia. After 10 min incubation, FM4-64 dye was rinsed from slides thoroughly before imaging fungal hyphae. Images were captured with a TCS SP8.

For host penetration assay, infected chickpea leaves (WT and $\Delta a r f-b a r$ ) were placed in $100 \%$ ethanol for $48 \mathrm{~h}$ to undergo bleaching for the complete removal of chlorophyll. Subsequently, leaves were incubated for $4 \mathrm{~h}$ in $10 \% \mathrm{KOH}$ at RT followed by washing $4-5$ times in phosphate buffer saline (PBS, $\mathrm{pH}$ 7.4). The processed leaves were then stained with chitin specific dye WGA-AF 488 (Invitrogen, USA). The leaf samples were rinsed in PBS (pH 7.5) before microscopic visualization. Confocal images were captured with a TCS SP5 confocal microscope.

For sub-cellular localization of ArCRZ1::EYFP under oxidative stress conditions, fungal conidia expressing chimeric protein were isolated and allowed to grow for $12 \mathrm{~h}$. The hyphae were exposed to $\mathrm{CaCl}_{2}$ (200 mM, Sigma-Aldrich, USA) for $1 \mathrm{~min}$ prior to microscopy. To assess the involvement of calcineurin in nuclear localization of ArCRZ1::EYFP, the hyphae were exposed to $5 \mu \mathrm{g} / \mu \mathrm{L}$ FK506 (Sigma-Aldrich, USA), $5 \mathrm{~min}$ prior to the addition of $\mathrm{CaCl}_{2}$. The confocal images were acquired with a TCS SP5.

For subcellular localization of ArCRZ1::EYFP during infection, susceptible plants were challenged with conidia expressing ArCRZ1::EYFP. The images were acquired after $48 \mathrm{~h}$ using TCS SP5.

Quantification and statistical analysis. Quantification analysis of relative fluorescent intensity, lesion size, radial diameter, and the distance of septa from the hyphal tip were analysed by Image/Fiji software. To calculate the significance of means/differences between two groups, Student's t-test and one-way ANOVA followed by Tukey test between multiple groups were performed using GraphPad Prism 6. Significance was accepted at $p<0.05$, as noted in the text of legends. Replicates are indicated in the legends.

Bioinformatic analysis. All the gene and protein sequences were acquired from NCBI server (http://www.ncbi.nlm.nih.gov). Multiple sequence alignment was performed by Praline search (http://www.ibi.vu.nl/programs/pralinewww/). Phylogenetic analysis was performed with MEGA7.0.21 software. Distinctive domain organization of the protein was determined by SMART search (http://smart.embl-heidelberg.de/). The putative TFs binding sites were identified by YEASTRACT-DISCOVERER Database (http://yeastract.com). The theoretical pI and molecular weight of the chimeric proteins were determined by Expasy compute $\mathrm{pI} / \mathrm{Mw}$ tool (http://web.expasy.org/cgi-bin/compute_pi/pi_tool). Protein IDs of protein sequences used in this study are: ArF-BAR: KZM20872.1, ArRab5: KZM19760.1, ArRab7: KZM26450.1, ArActin: KZM21342.1, ArWASP: KZM19192.1, ArCRZ1: KZM25117.1, and ArSNC1: KZM18806.1.

\section{Supporting information}

S1 Fig. Maximum likelihood phylogeny of F-BAR sequences. Sequences used: Ascochyta rabiei, Parastagonospora nodorum, Alternaria alternate, Bipolaris zeicola, Bipolaris victoriae, Bipolaris oryzae, Bipolaris sorokiniana, Bipolaris maydis, Sclerotinia sclerotiorum, Botrytis cinerea, Marssonina brunnea, Histoplasma capsulatum, Blastomyces dermatitidis, Blastomyces gilchristii, Emmonsia crescens, Paracoccidioides brasiliensis, Uncinocarpus reesii, Coccidioides immitis, Aspergillus nidulans, Aspergillus rambellii, Aspergillus ochraceoroseus, Aspergillus kawachii, Aspergillus lacticoffeatus, Aspergillus oryzae, Aspergillus flavus, Aspergillus parasiticus, Aspergillus nomius, Aspergillus terreus, Aspergillus fumigatus, Aspergillus lentulus, Aspergillu sclavatus, Aspergillus fischeri, Trichoderma reesei, Cordyceps confragosa, Fusarium oxysporum, Fusarium verticillioides, Fusarium fujikuroi, Fusarium graminearum, Fusarium pseudograminearum, Fusarium langsethiae, Neonectria ditissima, Metarhizium anisopliae, Metarhizium acridum, Pyricularia grisea, Pyricularia oryzae, Verticillium dahlia, Neurospora 
crassa, Drosophila melanogaster, Schizosaccharomyces pombe, Phytophthora sojae, Phytophthora graminis, Phytophthora striiformis, Ustilago maydis, Rhizopus delemar, Cryptococcus neoformans, Phytophthora infestans, Yarrowia lipolytica, Kluyveromyces lactis, Naumovozyma castellii, Saccharomyces cerevisiae, Candida glabrata, Eremothecium gossypii, Clavispora lusitaniae, Meyerozyma guilliermondii, Candida parapsilosis, Candida tropicalis, Candida dubliniensis, Candida albicans, Ganoderma lucidum, and Homo sapiens. The multiple sequence alignment of protein was performed by PROMALS3D software and the phylogeny was constructed using a software MEGA7.0.21. The bootstrap values, derived from 1000 iterations, validated the obtained phylogeny. (TIF)

S2 Fig. Conserved nature of ArF-BAR protein. Multiple sequence alignment showing the conservation of ArF-BAR protein with BZZ1p of S. cerevisiae, Cdc42-interacting protein 4 (CIP4) of Drosophila and Syndapin proteins of Drosophila. Colour code for sequence conservation varies from blue (least conserved) to red (highly conserved). The alignment of the protein is determined by Praline software using default parameters. The black box marks the presence of positively charged residues of F-BAR domain. Asterisk $\left(^{*}\right)$ represents the residues in $\mathrm{C} 1$ domain of protien Kinase C1 (PKC1), required for interaction with DAG.

(TIF)

S3 Fig. Expression and purification of ArF-BAR protein in E. coli. (A) His purification of bacterially expressed ArF-BAR protein. Analysis of the purification of recombinant ArF-BAR as shown by SDS-PAGE. (B) Intense tubular network in synthetic liposomes is formed after 30 min incubation with purified recombinant ArF-BAR protein. Inset showing the enlarged view of dense tubular network originating from a liposome. (C- D) His purification of ArF-BAR ${ }^{\text {mut1 }}$ and ArF-BAR ${ }^{\text {mut2 }}$. The protein was visualised by Coomassie Brilliant Blue staining. (UI- crude extract of un-induced samples after centrifugation; I- crude extract of induced samples after centrifugation; FT- flow-through fraction of the Nickel chelating resin column; W5- $5^{\text {th }}$ wash fraction of the Nickel chelating resin column; E1, E2 and E3- eluate fractions of the Nickel chelating resin column showing the purified ArF-BAR protein). Protein standards are shown (M) and their masses are indicated in $\mathrm{kDa}$.

S4 Fig. Southern hybridization confirmation of the successful replacement of $A r F-B A R$ gene with hygromycin cassette and complementation. (A) The schematic representation of A. rabiei knockout mutant generation by the homologous recombination approach to obtain targeted $A r F-B A R$ gene deletion mutants ( $\triangle a r f-b a r$ ). The bar represents the genomic region used to generate probe for Southern confirmation. (B) The representative Southern blot confirming successful $A r F-B A R$ gene deletion ( $\triangle a r f-b a r$ ), with a single integration of $h p h$ at replacement site. Along with the confirmation of $A r F-B A R$ complementation in $\triangle a r f-b a r$, followed successful generation of $\triangle a r f-b a r / A r F-B A R^{\text {mut1 }}$ and $\triangle a r f-b a r / A r F-B A R^{\text {mut2 }}$ complementation.

S5 Fig. The schematic representation of constructs used to generate $\Delta a r f-b a r$ mutant complemented strains. (A, B, C) Constructs used to generate different $\triangle a r f$-bar mutant complemented strains ( $\triangle a r f-b a r / A r F-B A R, \triangle a r f-b a r / A r F-B A R^{\text {mut1 }}$, and $\triangle a r f-b a r / A r F-B A R^{\text {mut2 }}$ ) under the control of native promoter of $A r F-B A R$ gene.

(TIF)

S6 Fig. Disease symptoms on AB susceptible chickpea plants after 10 days post inoculation. The susceptible plants inoculated with conidia of A. rabiei (WT), $\triangle a r f-b a r / A r F-B A R$, and 
$\triangle a r f-b a r / A r F-B A R^{\text {mut2 }}$, showed severe disease symptoms with the increasing duration after inoculation. $\triangle a r f$-bar and $\triangle a r f$-bar/ArF-BAR ${ }^{\text {mut1 }}$ challenged plants showed no or very mild symptoms after $10 \mathrm{dpi}$.

(TIF)

S7 Fig. Evaluation of chickpea host cells penetration by A. rabiei (WT) and $\Delta a r f-b a r$ strains growing hyphae. (A, B) Confocal images showing the depth of penetration 48 hpi by A. rabiei (WT) and $\triangle a r f-b a r$ strains, respectively, in AB susceptible chickpea leaves. Fungal hyphae were stained with WGA-488 for visualization, prior to microscopy. The $Z$-stacked images were acquired till $23 \mu \mathrm{m}$ depth, starting from the surface of the leaves. The image is the representation of maximum projections of all the Z-stacks. Scale bar $=5 \mu \mathrm{m}$. (C) The bar graph, representing mean and SD, shows the difference in ability to penetrate within the host by A. rabiei (WT) and $\Delta a r f-b a r$. The results were analysed using Student's t-test one tailed compared to its control $\left({ }^{*} p=0.0079\right)$.

S8 Fig. Growth inhibition in colony morphology of $\Delta a r f-b a r$ mutant and ArF-BAR complementation strains in the presence of various oxidative stresses. The A. rabiei (WT), $\Delta$ arfbar mutant and mutant complemented strains, observed 10 days after incubation at $22^{\circ} \mathrm{C}$. For oxidative stress, the PDA was supplemented with $250 \mu \mathrm{M}$ and $500 \mu \mathrm{M}$ menadione and $2 \mathrm{mM}$ $\mathrm{H}_{2} \mathrm{O}_{2}$. Strains of $\triangle a r f-b a r$ and $\triangle a r f-b a r / A r F-B A R^{\text {mut1 }}$ exhibited more growth inhibition towards oxidative stress condition as compared to WT. (TIF)

S9 Fig. Distribution of ArF-BAR::EYFP during in planta infection. Confocal micrographs showing the punctate distribution of ArF-BAR::EYFP during host infection. The representative image is the maximum intensity projection of all the Z-stack images with $0.5 \mu \mathrm{m}$ step size, acquired after 48 hpi of susceptible chickpea with fungal conidia expressing chimeric ArF-BAR::EYFP. Scale bar $=5 \mu \mathrm{m}(\mathrm{n}=12)$.

S10 Fig. Germination of fungal conidia of $A$. rabiei and $\Delta a r f-b a r$ on hydrophobic surface. Difference in growth pattern on hydrophobic surfaces was slightly observed and microscopic photographs (20X) were taken after $12 \mathrm{~h}$ of conidial spread. Scale bar $=50 \mu \mathrm{m}$.

S11 Fig. Homodimerization of F-BAR indicating conserved nature. Split-Ubiquitin based $\mathrm{Y} 2 \mathrm{H}$ system was used to determine the homodimerization between ArF-BAR proteins. Plates were photographed after $48 \mathrm{~h}$ of yeast growth. Strong positive interaction between two ArF-BAR proteins was reflected with the growth on $\mathrm{QDO}(\mathrm{SD} / \mathrm{-L} / \mathrm{-W} / \mathrm{-A} / \mathrm{-H})$ media and X-gal overlay assay to check the activation LacZ gene.

S12 Fig. F-BAR domain of ArF-BAR is not the direct member to interact. (A) The yeast two-hybrid result showing the positive interaction of ArF-BAR protein with ArWASp. (B) F-BAR domain (amino acids 1-325) of ArF-BAR protein [F-BAR $\left({ }_{\text {ArF-BAR }}\right)$ ] failed to interact with ArWASp in $\mathrm{Y} 2 \mathrm{H}$ system. Plates were photographed $48 \mathrm{~h}$ after yeast spotting. The interaction was confirmed by three independent replicates.

S13 Fig. Characterization of ArCRZ1 and confirmation of gene knockout and complementation. (A) Schematic representation of domain organisation of ArCRZ1 protein. (B) The 
schematic map showing the homologous recombination based knockout approach used for targeted $A r C R Z 1$ gene deletion mutant ( $\Delta c r z 1)$ strain generation. (C) Schematic representation of $\triangle a r c r z 1 / A r C R Z 1$ complementation construct under the native promoter of $A r C R Z 1$. The genomic region used to generate probe for confirmation by Southern hybridization is being highlighted. (D) The Southern blot result confirmed successful ArCRZ1 gene deletion ( $\triangle$ arcrz1), with single integration of $h p h$ at replacement site along with the complementation confirmation of $A r C R Z 1$ in $\triangle a r c r z 1$ mutant.

(TIF)

S1 Table. YEASTRACT result for the putative transcription factors bindings on the upstream regulatory sequences of $A r F-B A R$.

(DOCX)

S2 Table. Ascochyta rabiei strains used in this study.

(DOCX)

S3 Table. List of oligonucleotides used in this study.

(DOCX)

S1 Data. Supplementary Data.

(XLSX)

\section{Author Contributions}

Conceptualization: Vimlesh Kumar, Praveen Kumar Verma.

Data curation: Ankita Shree, Kunal Singh, Kamal Kumar, Vimlesh Kumar, Praveen Kumar Verma.

Formal analysis: Praveen Kumar Verma.

Funding acquisition: Praveen Kumar Verma.

Investigation: Manisha Sinha, Ankita Shree, Kunal Singh, Shreenivas Kumar Singh.

Methodology: Manisha Sinha, Ankita Shree, Kunal Singh, Shreenivas Kumar Singh, Praveen Kumar Verma.

Project administration: Praveen Kumar Verma.

Resources: Kamal Kumar, Shreenivas Kumar Singh.

Supervision: Kunal Singh, Kamal Kumar, Vimlesh Kumar, Praveen Kumar Verma.

Validation: Manisha Sinha, Ankita Shree.

Visualization: Manisha Sinha, Ankita Shree, Praveen Kumar Verma.

Writing - original draft: Manisha Sinha, Ankita Shree, Vimlesh Kumar, Praveen Kumar Verma.

Writing - review \& editing: Ankita Shree, Kamal Kumar, Praveen Kumar Verma.

\section{References}

1. Takeshita N, Evangelinos M, Zhou L, Serizawa T, Somera-Fajardo RA, Lu L, et al. Pulses of $\mathrm{Ca}^{2+}$ coordinate actin assembly and exocytosis for stepwise cell extension. Proc Natl Acad Sci U S A. 2017; 114: 5701-5706. https://doi.org/10.1073/pnas.1700204114 PMID: 28507141 
2. Riquelme M, Aguirre J, Bartnicki-García S, Braus GH, Feldbrügge M, Fleig U, et al. Fungal morphogenesis, from the polarized growth of hyphae to complex reproduction and infection structures. Microbiol Mol Biol Rev. 2018; 82: e00068-17. https://doi.org/10.1128/MMBR.00068-17 PMID: 29643171

3. Etxebeste $\mathrm{O}$, Espeso EA. Neurons show the path: tip-to-nucleus communication in filamentous fungal development and pathogenesis. FEMS Microbiol Rev. 2016; 40: 610-624. https://doi.org/10.1093/ femsre/fuw021 PMID: 27587717

4. Bielska E, Higuchi Y, Schuster M, Steinberg N, Kilaru S, Talbot NJ, et al. Long-distance endosome trafficking drives fungal effector production during plant infection. Nat Commun. 2014; 5: 5097. https://doi. org/10.1038/ncomms6097 PMID: 25283249

5. Higuchi $\mathrm{Y}$, Arioka M, Kitamoto K. Endocytic recycling at the tip region in the filamentous fungus AspergilIus oryzae. Commun Integr Biol. 2009; 2: 327-328. https://doi.org/10.4161/cib.2.4.8385 PMID: 19721880

6. Higuchi Y, Shoji JY, Arioka M, Kitamoto K. Endocytosis is crucial for cell polarity and apical membrane recycling in the filamentous fungus Aspergillus oryzae. Eukaryot Cell. 2009; 8: 37-46. https://doi.org/ 10.1128/EC.00207-08 PMID: 19028995

7. Takeshita N. Coordinated process of polarized growth in filamentous fungi. Biosci Biotechnol Biochem. 2016; 80: 1693-1699. https://doi.org/10.1080/09168451.2016.1179092 PMID: 27121747

8. Takeshita N, Higashitsuji Y, Konzack S, Fischer R. Apical sterol-rich membranes are essential for localizing cell end markers that determine growth directionality in the filamentous fungus Aspergillus nidulans. Mol Biol Cell. 2008; 19: 339-351. https://doi.org/10.1091/mbc.e07-06-0523 PMID: 18003978

9. Rao Y, Rückert C, Saenger W, Haucke V. The early steps of endocytosis: from cargo selection to membrane deformation. Eur J Cell Biol. 2012; 91: 226-233. https://doi.org/10.1016/j.ejcb.2011.02.004 PMID: 21458101

10. Frost A, Perera R, Roux A, Spasov K, Destaing O, Egelman EH, et al. Structural basis of membrane invagination by F-BAR domains. Cell. 2008; 132: 807-817. https://doi.org/10.1016/j.cell.2007.12.041 PMID: 18329367

11. Habermann B. The BAR-domain family of proteins: a case of bending and binding? EMBO Rep. 2004; 5: 250-255. https://doi.org/10.1038/sj.embor.7400105 PMID: 14993925

12. Krauss M, Haucke V. A novel twist in membrane dePHormation. Dev Cell. 2014; 31: 3-4. https://doi. org/10.1016/j.devcel.2014.09.016 PMID: 25313957

13. Zimmerberg J, Kozlov MM. How proteins produce cellular membrane curvature. Nat Rev Mol Cell Biol. 2006; 7: 9-19. https://doi.org/10.1038/nrm1784 PMID: 16365634

14. Saarikangas J, Zhao H, Pykäläinen A, Laurinmäki P, Mattila PK, Kinnunen PKJ, et al. Molecular mechanisms of membrane deformation by I-BAR domain proteins. Curr Biol. 2009; 19: 95-107. https://doi.org/ 10.1016/j.cub.2008.12.029 PMID: 19150238

15. Fuchs U, Hause G, Schuchardt I, Steinberg G. Endocytosis is essential for pathogenic development in the corn smut fungus Ustilago maydis. Plant Cell. 2006; 18: 2066-2081. https://doi.org/10.1105/tpc. 105.039388 PMID: 16798890

16. Böhmer C, Ripp C, Bölker M. The germinal centre kinase Don3 triggers the dynamic rearrangement of higher-order septin structures during cytokinesis in Ustilago maydis. Mol Microbiol. 2009; 74: 14841496. https://doi.org/10.1111/j.1365-2958.2009.06948.x PMID: 19906182

17. Lin L, Chen X, Shabbir A, Chen S, Chen X, Wang Z, et al. A putative N-BAR-domain protein is crucially required for the development of hyphae tip appressorium-like structure and its plant infection in Magnaporthe oryzae. Phytopathol Res. 2019; 1: 32. https://doi.org/10.1186/s42483-019-0038-2

18. Dagdas YF, Yoshino K, Dagdas G, Ryder LS, Bielska E, Steinberg G, et al. Septin-mediated plant cell invasion by the rice blast fungus, Magnaporthe oryzae. Science. 2012; 336: 1590-1595. https://doi.org/ 10.1126/science.1222934 PMID: 22723425

19. Soulard A, Lechler T, Spiridonov V, Shevchenko A, Shevchenko A, Li R, et al. Saccharomyces cerevisiae Bzz1p is implicated with type I myosins in actin patch polarization and is able to recruit actin-polymerizing machinery in vitro. Mol Cell Biol. 2002; 22: 7889-7906. https://doi.org/10.1128/mcb.22.22. 7889-7906.2002 PMID: 12391157

20. Fricke R, Gohl C, Dharmalingam E, Grevelhörster A, Zahedi B, Harden N, et al. Drosophila Cip4/Toca-1 integrates membrane trafficking and actin dynamics through WASP and SCAR/WAVE. Curr Biol. 2009; 19: 1429-1437. https://doi.org/10.1016/j.cub.2009.07.058 PMID: 19716703

21. Arasada R, Pollard TD. Distinct roles for F-BAR proteins Cdc15p and Bzz1p in actin polymerization at sites of endocytosis in fission yeast. Curr Biol. 2011; 21: 1450-1459. https://doi.org/10.1016/j.cub.2011. 07.046 PMID: 21885283

22. Kumar K, Purayannur S, Kaladhar VC, Parida SK, Verma PK. mQTL-seq and classical mapping implicates the role of an AT-HOOK MOTIF CONTAINING NUCLEAR LOCALIZED (AHL) family gene in 
Ascochyta blight resistance of chickpea. Plant Cell Environ. 2018; 41: 2128-2140. https://doi.org/10. 1111/pce.13177 PMID: 29492990

23. Pandey BK, Singh US, Chaube HS. Mode of infection of Ascochyta blight of chickpea caused by Ascochyta rabiei. J Phytopathol. 1987; 119: 88-93. https://doi.org/10.1111/j.1439-0434.1987.tb04387.x

24. Ilarslan H, Dolar FS. Histological and ultrastructural changes in leaves and stems of resistant and susceptible chickpea cultivars to Ascochyta rabiei. J Phytopathol. 2002; 150: 340-348. https://doi.org/10. 1046/j.1439-0434.2002.00763.x

25. Verma S, Gazara RK, Nizam S, Parween S, Chattopadhyay D, Verma PK. Draft genome sequencing and secretome analysis of fungal phytopathogen Ascochyta rabiei provides insight into the necrotrophic effector repertoire. Sci Rep. 2016; 6: 24638. https://doi.org/10.1038/srep24638 PMID: 27091329

26. Singh K, Nizam S, Sinha M, Verma PK. Comparative transcriptome analysis of the necrotrophic fungus Ascochyta rabiei during oxidative stress: Insight for fungal survival in the host plant. PLoS One. 2012; 7: e33128. https://doi.org/10.1371/journal.pone.0033128 PMID: 22427966

27. Nizam S, Singh K, Verma PK. Expression of the fluorescent proteins DsRed and EGFP to visualize early events of colonization of the chickpea blight fungus Ascochyta rabiei. Curr Genet. 2010; 56: 391399. https://doi.org/10.1007/s00294-010-0305-3 PMID: 20461519

28. Tsujita K, Suetsugu S, Sasaki N, Furutani M, Oikawa T, Takenawa T. Coordination between the actin cytoskeleton and membrane deformation by a novel membrane tubulation domain of $\mathrm{PCH}$ proteins is involved in endocytosis. J Cell Biol. 2006; 172: 269-279 https://doi.org/10.1083/jcb.200508091 PMID: 16418535

29. Kumar V, Fricke R, Bhar D, Reddy-Alla S, Krishnan KS, Bogdan S, et al. Syndapin promotes formation of a postsynaptic membrane system in Drosophila. Mol Biol Cell. 2009; 20: 2254-2264. https://doi.org/ 10.1091/mbc.e08-10-1072 PMID: 19244343

30. Heung LJ, Luberto C, Plowden A, Hannun YA, Del Poeta M. The sphingolipid pathway regulates Pkc1 through the formation of diacylglycerol in Cryptococcus neoformans. J Biol Chem. 2004; 279:2114421153. https://doi.org/10.1074/jbc.M312995200 PMID: 15014071

31. Lanver D, Mendoza-Mendoza A, Brachmann A, Kahmann R. Sho1 and Msb2-related proteins regulate appressorium development in the smut fungus Ustilago maydis. Plant Cell. 2010; 22:2085-2101. https://doi.org/10.1105/tpc.109.073734 PMID: 20587773

32. Valkonen M, Kalkman ER, Saloheimo M, Penttilä M, Read ND, Duncan RR. Spatially segregated SNARE protein interactions in living fungal cells. J Biol Chem. 2007; 282:22775-22785. https://doi.org/ 10.1074/jbc.M700916200 PMID: 17553800

33. Li YB, Xu R, Liu C, Shen N, Han LB, Tang D. Magnaporthe oryzae fimbrin organizes actin networks in the hyphal tip during polar growth and pathogenesis. PLoS Pathog. 2020; 16:e1008437. https://doi.org/ 10.1371/journal.ppat.1008437 PMID: 32176741

34. Giraldo MC, Dagdas YF, Gupta YK, et al. Two distinct secretion systems facilitate tissue invasion by the rice blast fungus Magnaporthe oryzae. Nat Commun. 2013; 4:1996. https://doi.org/10.1038/ ncomms2996 PMID: 23774898

35. Qualmann B, Koch D, Kessels MM. Let's go bananas: Revisiting the endocytic BAR code. EMBO J. 2011; 30: 3501-3515. https://doi.org/10.1038/emboj.2011.266 PMID: 21878992

36. Fischer-Parton S, Parton RM, Hickey PC, Dijksterhuis J, Atkinson HA, Read ND. Confocal microscopy of FM4-64 as a tool for analysing endocytosis and vesicle trafficking in living fungal hyphae. J Microsc. 2000; 198: 246-259. https://doi.org/10.1046/j.1365-2818.2000.00708.x PMID: 10849201

37. Zeigerer A, Gilleron J, Bogorad RL, Marsico G, Nonaka H, Seifert S, et al. Rab5 is necessary for the biogenesis of the endolysosomal system in vivo. Nature. 2012; 485: 465-470. https://doi.org/10.1038/ nature11133 PMID: 22622570

38. Steinberg Endocytosis and early endosome motility in filamentous fungi. Curr Opin Microbiol. 2014; 20: 10-18. https://doi.org/10.1016/j.mib.2014.04.001 PMID: 24835422

39. Almeida-Souza L, Frank RAW, García-Nafría J, Colussi A, Gunawardana N, Johnson CM, et al. A flat BAR protein promotes actin polymerization at the base of clathrin-coated pits. Cell. 2018; 174: 325337.e14. https://doi.org/10.1016/j.cell.2018.05.020 PMID: 29887380

40. Berepiki A, Lichius A, Read ND. Actin organization and dynamics in filamentous fungi. Nat Rev Microbiol. 2011; 9: 876-887. https://doi.org/10.1038/nrmicro2666 PMID: 22048737

41. Riedl J, Crevenna AH, Kessenbrock K, Yu JH, Neukirchen D, et al. Lifeact: a versatile marker to visualize F-actin. Nat Methods. 2008; 5: 605-607. https://doi.org/10.1038/nmeth.1220 PMID: 18536722

42. Monteiro PT, Oliveira J, Pais $P$, Antunes M, Palma M, Cavalheiro M, et al. YEASTRACT+: a portal for cross-species comparative genomics of transcription regulation in yeasts. Nucleic Acids Res. 2020; 48: D642-D649. https://doi.org/10.1093/nar/gkz859 PMID: 31586406 
43. Park HS, Lee SC, Cardenas ME, Heitman J. Calcium-calmodulin-calcineurin signaling: a globally conserved virulence cascade in eukaryotic microbial pathogens. Cell Host Microbe. 2019; 26: 453-462. https://doi.org/10.1016/j.chom.2019.08.004 PMID: 31600499

44. Hernández-Ortiz $P$, Espeso EA. Spatiotemporal dynamics of the calcineurin target CrzA. Cell Signal. 2017; 29: 168-180. https://doi.org/10.1016/j.cellsig.2016.11.005 PMID: 27832964

45. Yoshimoto H, Saltsman K, Gasch AP, Li HX, Ogawa N, Botstein D, et al. Genome-wide analysis of gene expression regulated by the calcineurin/Crz1p signaling pathway in Saccharomyces cerevisiae. J Biol Chem. 2002; 277: 31079-31088. https://doi.org/10.1074/jbc.M202718200 PMID: 12058033

46. Li X, Gao C, Li L, Liu M, Yin Z, Zhang H, et al. MoEnd3 regulates appressorium formation and virulence through mediating endocytosis in rice blast fungus Magnaporthe oryzae. PLoS Pathog. 2017; 13: e1006449. https://doi.org/10.1371/journal.ppat.1006449 PMID: 28628655

47. Leibfried A, Fricke R, Morgan MJ, Bogdan S, Bellaiche Y. Drosophila Cip4 and WASp define a branch of the Cdc42-Par6-aPKC pathway regulating E-cadherin endocytosis. Curr Biol. 2008; 18: 1639-1648. https://doi.org/10.1016/j.cub.2008.09.063 PMID: 18976911

48. Grove SN, Bracker CE. Protoplasmic organization of hyphal tips among fungi: vesicles and Spitzenkörper. J Bacteriol. 1970; 104: 989-1009. https://doi.org/10.1128/JB.104.2.989-1009.1970 PMID: 4099103

49. Peñalva MÁ. Endocytosis in filamentous fungi: Cinderella gets her reward. Curr Opin Microbiol. 2010; 13: 684-692. https://doi.org/10.1016/j.mib.2010.09.005 PMID: 20920884

50. Lo Presti L, Lanver D, Schweizer G, Tanaka S, Liang L, Tollot M, et al. Fungal effectors and plant susceptibility. Annu Rev Plant Biol. 2015; 66: 513-545. https://doi.org/10.1146/annurev-arplant-043014114623 PMID: 25923844

51. Singh $Y$, Nair AM, Verma PK. Surviving the odds: from perception to survival of fungal phytopathogens under host-generated oxidative burst. Plant Commun. 2021. https://doi.org/10.1016/j.xplc.2021.100142

52. de Castro PA, Colabardini AC, Manfiolli AO, Chiaratto J, Silva LP, Mattos EC, et al. Aspergillus fumigatus calcium-responsive transcription factors regulate cell wall architecture promoting stress tolerance, virulence and caspofungin resistance. PLoS Genet. 2019; 15: e1008551. https://doi.org/10.1371/ journal.pgen.1008551 PMID: 31887136

53. Harris SD. Septum formation in Aspergillus nidulans. Curr Opin Microbiol. 2001; 4: 736-739. https://doi. org/10.1016/s1369-5274(01)00276-4 PMID: 11731327

54. Frost $A$, Unger VM, De Camilli $P$. The BAR domain superfamily: membrane-molding macromolecules. Cell. 2009; 137: 191-196. https://doi.org/10.1016/j.cell.2009.04.010 PMID: 19379681

55. Delgado-Álvarez DL, Bartnicki-García S, Seiler S, Mouriño-Pérez RR. Septum development in Neurospora crassa: The septal actomyosin tangle. PLoS One. 2014; 9: e96744. https://doi.org/10.1371/ journal.pone.0096744 PMID: 24800890

56. Guertin DA, Trautmann S, McCollum D. Cytokinesis in eukaryotes. Microbiol Mol Biol Rev. 2002; 66: 155-178. https://doi.org/10.1128/mmbr.66.2.155-178.2002 PMID: 12040122

57. Wu JQ, Kuhn JR, Kovar DR, Pollard TD. Spatial and temporal pathway for assembly and constriction of the contractile ring in fission yeast cytokinesis. Dev Cell. 2003; 5: 723-734. https://doi.org/10.1016/ s1534-5807(03)00324-1 PMID: 14602073

58. Martín-García R, Arribas V, Coll PM, Pinar M, Viana RA, Rincón SA, et al. Paxillin-mediated recruitment of calcineurin to the contractile ring is required for the correct progression of cytokinesis in fission yeast. Cell Rep. 2018; 25: 772-783.e4. https://doi.org/10.1016/j.celrep.2018.09.062 PMID: 30332655

59. Lamb CJ, Lawton MA, Dron M, Dixon RA. Signals and transduction mechanisms for activation of plant defenses against microbial attack. Cell. 1989; 56: 215-224. https://doi.org/10.1016/0092-8674(89) 90894-5 PMID: 2643475

60. Livak KJ, Schmittgen TD. Analysis of relative gene expression data using real-time quantitative PCR and the 2- $\Delta \Delta$ CT method. Methods. 2001; 25: 402-408. https://doi.org/10.1006/meth.2001.1262 PMID: 11846609

61. Talbot NJ, Ebbole DJ, Hamer JE. Identification and characterization of MPG1, a gene involved in pathogenicity from the rice blast fungus Magnaporthe grisea. Plant Cell. 1993; 5: 1575-1590 https://doi.org/ 10.1105/tpc.5.11.1575 PMID: 8312740 